\title{
活性羰基化合物苂光探针的研究进展
}

\author{
陈炎胡奥晗杨凌毅李早英严 琨* \\ (武汉大学化学与分子科学学院 武汉 430072)
}

\begin{abstract}
摘要 活性羰基化合物一直以来被人们称为致癌物和人类毒物, 当其浓度升高时, 会导致多种疾病, 因此, 设计开发 用于选择性识别和高灵敏检测生物体内的活性羰基化合物的技术具有十分重要的意义. 近年来，苂光探针具有操作简 单、灵敏度高、选择性好，响应时间短以及能实现实时检测等优势，在活性羰基化合物及其他活性物种如活性氧、活 性氮、次氯酸以及硫醇等物质的检测方面获得了快速发展. 鉴于活性羰基化合物荧光探针综述方面的报道很少，从探 针分子与待测物之间发生的反应类别进行归纳和总结，概括了甲醛、甲基乙二醛、丙二醛以及丙烯醛等活性羰基化合 物的苂光探针, 并从设计理念、识别机理以及应用等方面进行了描述，还对活性羰基化合物苂光探针的设计和应用前 景提出了展望.
\end{abstract}

关键词 活性羰基化合物；荧光探针；分子设计；甲醛；甲基乙二醛

\section{Recent Progress in Fluorescent Probe for the Detection of Reactive Carbonyl Species}

\author{
Chen, Yi Hu, Aohan Yang, Lingyi $\quad$ Li, Zaoying $\quad$ Yan, Kun* \\ (College of Chemistry and Molecular Sciences, Wuhan University, Wuhan 430072)
}

\begin{abstract}
Reactive carbonyl species (RCS) have long been known as carcinogens and human poison. Elevated levels of RCS have also been associated with pathologies. In order to elucidate these roles of RCS, the design and development technology for selective and sensitive detection to RCS in vivo are crucial. In rencent years, fluorescent probes for detecting reactive carbonyl species, reactive oxygen, reactive nitrogen, hypochlorous acid and thiols have been developed due to various advantages such as simplicity of operation, high sensitivity and selectivity, response quickly and real-time tracking in living cells. Few reviews have been made for detecting reactive carbonyl species. Therefore, in this review, a general overview of the reaction-based fluoresccent in the selected recent research is provided involving design, recognition mechanism and application of fluorescent probes for detecting reactive carbonyl species of formaldehyde, methylglyoxal, malondialdehyde, acrolein and so on. Finally, the prospect to design and applications of probes for reactive carbonyl species is given.

Keywords reactive carbonyl species; fluorescent probe; molecular design; formaldehyde; methylglyoxal
\end{abstract}

活性羰基化合物(Reactive carbonyl species, RCS)一 直以来被人们称为致癌物和人类毒物，它的来源分为两 种: 第一, 食物的加工过程; 第二, 生物体内碳水化合 物、脂质以及氨基酸等物质氧化后的副产物 ${ }^{[1]}$. 生物体 内的活性羰基化合物是一类包含一个或多个羰基基团 的高活性亲电化合物, 主要包括甲醛(FA)、甲基乙二醛 $(M G O)$ 、丙二醛(MDA)、丙烯醛、丙醛、乙二醛、乙醛、 4-羊基壬烯醛、草酰乙酸、丙酮酸等 ${ }^{[2]}$. 研究表明, 正常 情况下, 生物体内活性羰基化合物的浓度维持在一个正
常的范围之内，当其浓度升高时，会导致多种疾病，如 各种癌症、神经紊乱、糖尿病以及心脏病等 ${ }^{[3]}$. 考虑到 生物体内羰基化合物的浓度与人体健康的重大关系，探 研一种高效的用来检测活性羰基化合物的技术显得至 关重要.

传统的用来检测活性羰基化合物的方法主要包括 4 种, 即辐射测定法、气相色谱法、高效液相色谱法和比 色法等 ${ }^{[4]}$. 同样地, 它们能提供较高的灵敏性和选择性, 但是存在一些不足, 如样品处理较麻烦, 不能保证样品

\footnotetext{
* Corresponding author. E-mail: kyan@whu.edu.cn Received March 24, 2017; revised April 16, 2017; published online May 4, 2017.
}

Project supported by the National Natural Science Foundation of China (No. 21401144) and the Natural Science Foundation of Hubei Province (No. 2013CFB236).

国家自然科学基金(No. 21401144)及湖北省自然科学基金(No. 2013CFB236)资助项目. 
的完整性，也不能用于生物体内 RCS 的实时探测. 对于 活性羰基化合物的检测，传统的检测方法都存在一定的 缺陷和不足, 而近期发展起来的荧光探针的检测方法常 用于检测生物体内的一些物质, 如硫醇 ${ }^{[5]}$ 、金属离子 ${ }^{[6]}$ 、 活性氧物种 ${ }^{[7]}$ 以及活性氮物种 ${ }^{[8]}$ 等, 具有操作简单、高灵 敏性、高选择性、响应时间短而且检测过程对样品没有 破坏, 对活细胞造成的伤害也较小, 能实现生物体内的 实时监测等优点. 至今为止, 苂光探针在生物体内的成 像研究主要分为三个阶段, 即细胞内成像阶段 ${ }^{[9]}$, 活体 组织成像阶段 ${ }^{[10]}$ 以及动物体内活体成像阶段 ${ }^{[11]}$, 这三 个阶段分布进行, 推进着荧光探针向不同领域的快速发 展. 目前苂光探针在生物体内的成像研究主要集中在活 细胞成像阶段, 并且近年来相继报道一些靶向定位到某 个特定的细胞器, 如线粒体 ${ }^{[12]}$ 、溶酶体 ${ }^{[13]}$ 和高尔基体 ${ }^{[14]}$ 等的苂光探针分子, 引起了科研工作者的研究兴趣.

关于活性羰基化合物的苂光探针方面的研究一直 是科研工作者的重点研究方向. 关于这类物质在荧光探 针方面的报道也不断增加, 但是, 关于活性羰基化合物 苂光探针研究进展方面的综述甚少. 本文通过探针分子 与待测物之间发生的化学反应的类型, 总结了常见的活 性羰基化合物如甲醛(FA)、甲基乙二醛(MGO)、丙二醛 (MDA)、丙烯醉等物质的苂光探针, 并且对一些苂光探 针的设计理念、识别机理以及应用进行了总结和归纳.

\section{1 检测甲醛(FA)的荧光探针}

甲醛，作为结构最简单的醛类, 是一种具有高度反 应活性的岸基物种. 生物体主要通过脱甲基酶和氧化酶 在体内产生甲醛 ${ }^{[15]}$, 当生物体内甲醛的浓度发生异常 时, 会引发癌症 ${ }^{[16]}$ 、神经性疾病 ${ }^{[17]}$ 、糖尿病、肝病以及 心脏病 ${ }^{[18]}$ 等，因此，设计合成用于快速、高效、实时检 测生物体内甲醛的探针, 在探究甲醛关于生理、病理的
研究以及疾病诊断和控制等方面的应用意义重大。目 前, 关于甲醛苂光探针的设计主要采用的反应机理有 Aza-cope 重排、 $\mathrm{NHNH}_{2}$ 以及 $\mathrm{NH}_{2}$ 与甲醛的反应、金属 配合物与甲醛的反应，以及其它反应类型五大类.

\section{1 基于探针与甲醛发生 Aza-cope 反应的探针}

Aza-cope 重排反应作为有机化学中合成复杂有机 化合物的重要方法, 它的重排机理如 Scheme 1 所示, 含 氮的 1,5-二烯烃会发生 cope 重排一样的[3,3]-键重排, 重排之后连续进行 Mannich 反应, 这种连续的反应被称 为 Aza-cope 重排反应 ${ }^{[19]}$.

2015 年, Chang 课题组 ${ }^{[20]}$ 设计出了一种基于甲醛诱 导 Aza-cope 重排反应的 “Turn-on” 型荧光探针 1, 用于 检测细胞内的甲醛. 该探针的识别机理如 Scheme 2 所 示. 探针 1 以氨甲基硅罗丹明染料为母体，分子内有一 个关闭的五元环, 表现出较弱的荧光, 与甲醛发生反应 后，探针 1 发生 Aza-cope 重排，最终发生水解，得到一 种具有较强苂光的目标产物, 能够达到对甲醛分子的可 视化识别. 在磷酸盐缓冲溶液 $(\mathrm{pH}=7.4)$ 缓冲溶液中, 探 针 $\mathbf{1}$ 表现出良好的水溶性以及微弱的荧光, 苂光量子产 率 $(\Phi)$ 为 0.36 , 加入甲醛之后, 在 $645 \mathrm{~nm}$ 处可以看到荧 光强度增强, 这是因为甲醛的加入导致五元环的开裂, 从而生成荧光较强的目标产物. 探针 1 对甲醛分子的检

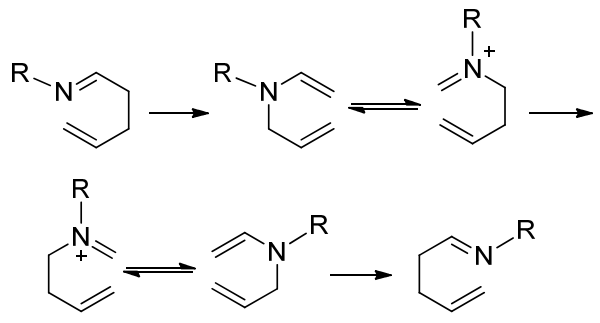

图式 1 Aza-cope 重排的机理 Scheme 1 Mechanism of aza-cope reaction

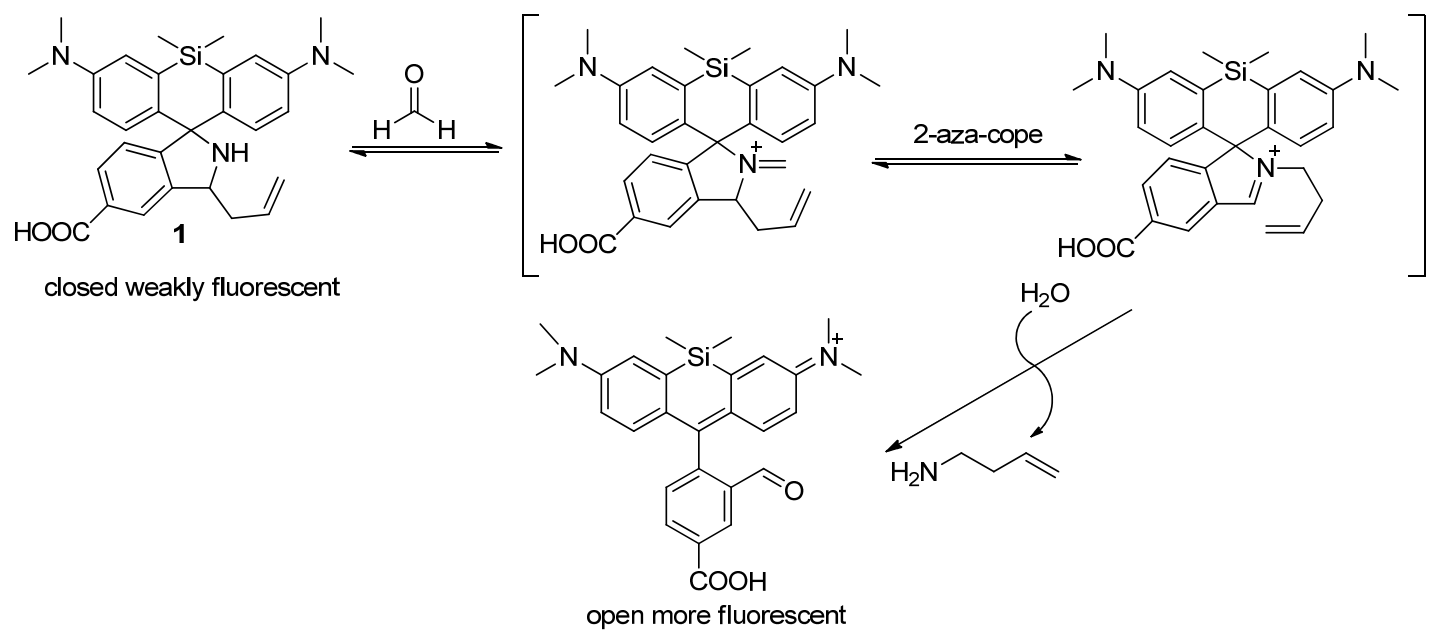

图式 2 探针 1 的结构及其对甲醛的响应机理

Scheme 2 Structure of probe 1 and its responding reaction of formaldehyde 
测具有高选择性和高灵敏性，此外，探针 1 不仅可以检 测外源性甲醛，同时可以检测人体肾上腺细胞 (HEK293T) 等细胞中赖氨酸特异性蛋白酶去甲基化产 生的内源性甲醛. 因此, 探针 1 在生物体内检测甲醛分 子具有潜在的应用价值, 为生物体在遗传, 衰老和疾病 等生理过程中探测活性羰基化合物提供了一种研究手 段.

同样地, Chan 课题组 ${ }^{[21]}$ 基于硅罗丹明为骨架，也设 计合成了一个 “Turn-on” 型苂光探针 2, 用于检测细胞 内的甲醛(如 Scheme 3 所示). 探针 2 由三部分组成: 黑 色部分为苂光团, 蓝色部分为甲醛分子的识别基团, 基 于 Aza-cope 重排反应, 红色部分则代表着苂光猝灭基 团. 苂光猝灭基团的引入, 使分子中存在光诱导电子转 移过程(d-PET), 使得探针 $\mathbf{2}$ 本身没有显示出荧光信号, 与甲醛发生 Aza-cope 重排反应之后, 水解得到的目标 产物中猝灭基团离去, a-PET 过程被中断, 表现出较强 的荧光. 探针 2 具有良好的光物理性质，对甲醛分子的 检测表现出高选择性和高灵敏性, 探针 2 可用于人胚肾 细胞(HEK293TN)和脑神经瘤细胞(Neuroscreen-1)活细 胞成像. 除此之外, 探针 2 显示出优异的光稳定性, 作 者进行了时间推移成像实验并且可以实时捕获探针 $\mathbf{2}$ 和 甲醛之间的反应. 因此, 探针 2 为甲醛在活细胞正常以 及病理过程方面的研究提供了便利.
2016 年, Zeng 和 Sheng 等 ${ }^{[22]}$ 报道了基于荎为母体的 苂光探针 3(如 Eq. 1 所示). 探针 3 在 DMF-PBS 缓冲溶 液 $(V: V=1: 4, \mathrm{pH}=7.4)$ 中, 加入甲醛分子之后, 溶液 颜色发生改变, 从无色变为黄色, 能达到肉眼识别, 并 且在 $513 \mathrm{~nm}$ 处的荧光强度升高, 这主要是由于加入甲 醛分子之后，发生 Aza-cope 重排反应，生成的目标产物 分子内出现 push-pull (推拉)电子效应，导致分子内电荷 转移, 从而引起明显的苂光变化. 探针 3 在 $\mathrm{pH}=3 \sim$ 10.5 范围内对甲醛分子的选择性较好, 最小的检测限浓 度为 $0.57 \mu \mathrm{mol} / \mathrm{L}$. 探针 3 能用于食物里面甲醛的定量检 测, 也能用于 Hela 细胞中甲醛分子的活细胞成像.

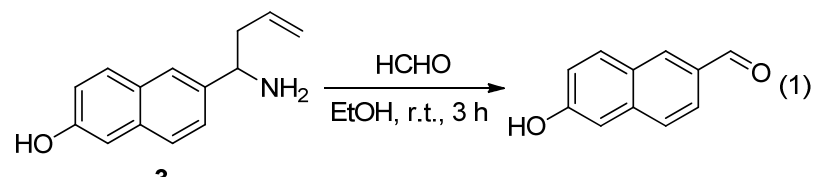

Lin 课题组 ${ }^{[23]}$ 报道了一种基于 6 -羟基䒺为苂光团的 甲醛比率型苂光探针 4 (如 Scheme 4 所示), 该探针在磷 酸盐缓冲溶液 $(25 \mathrm{mmol} / \mathrm{L}, \mathrm{pH}=7.4$, 含 $1 \%$ 的丙酮 $)$ 中, 加入甲醛之后，探针 4 在 $359 \mathrm{~nm}$ 处的苂光强度呈现降低 的趋势, $451 \mathrm{~nm}$ 处的苂光强度显示增加, $I_{451} / I_{359}$ 增加 53.2 倍，实现对甲醛的识别. 该探针在 $\mathrm{pH}=7.4$ 的磷酸 盐缓冲溶液中的检测限为 $5.96 \times 10^{-5} \mathrm{~mol} / \mathrm{L}$, 在 $\mathrm{pH}=4.5$<smiles>C=CCC(Nc1ccc([N+](=O)[O-])cc1)c1cn(C)c2cc(C)c(C3=C4C=CC(=O)C=C4[Si](C)(C)c4c3cc3c5c4CCCN5CCC3)cc12</smiles><smiles>C/C=C\C(C)=C/Cc1cc2c3c(c1SC)CCCN3CCC2</smiles><smiles>C=CCC(C1=CN(C)C2=CC(C)=C(C3=C4C=CC(=O)C=C4[Si](C)(C)/C3=C/C)C(=C)C1=C2)[N+](=C)c1ccc(N)cc1</smiles><smiles>CC(C)(C)C(C)(C)C</smiles>

Highly fluorescent

图式 3 探针 2 的结构及其对甲醛的识别机制

Scheme 3 Structure of the probe 2 and its detection strategy of formaldehyde<smiles>C=CCC(N=[N+]=C)c1ccc2cc(O)ccc2c1</smiles>

图式 4 探针 4 对甲醛的响应机理

Scheme 4 Response mechanism of 4 to formaldehyde 
的 PBS 缓冲溶液中低至 $1.87 \times 10^{-5} \mathrm{~mol} / \mathrm{L}$. 作者用理论 计算验证了探针 4 与甲醛分子的作用机理, 并且把探针 4 用于 HeLa 细胞的成像, 结果表明探针 $\mathbf{4}$ 能用于水溶液 以及生物样品中甲醛分子的检测.

在活细胞成像方面, 双光子苂光探针的优点十分显 著, 双光子荧光成像主要通过双光子显微镜来完成. 科 学实验证实, 设计合成一个好的双光子荧光探针可以从 以下几个方面着手, 即在近红外区域拥有大的双光子吸 收截面、在水中具有较好的溶解性、对光稳定以及能快 速特异性识别某些受体分子 ${ }^{[24]}$. Yuan 和 Zhang 等 ${ }^{[25]}$ 设计 合成了一种双光子荧光探针 5(如 Scheme 5 所示), 以䒬 衍生物为苂光团, 硝基苯为荧光猝灭基团, 胺为甲醛的 识别基团. 该探针在 PBS-DMSO $(V: V=19: 1, \mathrm{pH}=$ 7.4)缓冲溶液中表现出较弱的苂光, 加入甲醛分子, $3 \mathrm{~h}$ 之后, $526 \mathrm{~nm}$ 处的苂光增强 95 倍, 这主要是由于与甲醛 反应生成的目标产物, 具有 D- $\pi$-A 结构, 从而表现出一 种 turn-on 型苂光反应. 该探针在 $\mathrm{pH}=2 \sim 10$ 范围内对 甲醛分子具有较高的选择性, 检测限低至 $0.20 \mu \mathrm{mol} / \mathrm{L}$. 除此之外，探针 5 具有较低的细胞毒性以及良好的光稳 定性, 可以用于内源性以及外源性生物体内甲醛的检测 和研究, 因此, 探针 5 在生物体内检测甲醛分子具有广 泛的应用价值.

同样地, Sun 和 Zhu 课题组 ${ }^{[26]}$ 也报道了另外一种新 型双光子荧光探针 6 (如 Eq. 2 所示), 该探针以具有较大 的斯托克位移以及较好的光稳定性的双光子荧光发射 的 4-羟基 1,8-菜二甲酰亚胺 ${ }^{[27]}$ 为母体, 通过 Duff 反应在 羟基的邻位引入醛基, 进而引入甲醛的识别基团. 探针 6 在 PBS 缓冲溶液 $(10 \mathrm{mmol} / \mathrm{L}, \mathrm{pH}=7.4$, 含 $0.5 \%$ 的二 甲亚砜)中, 在 $448 \mathrm{~nm}$ 处有较强的紫外-可见吸收峰, 荧 光发射波长为 $550 \mathrm{~nm}, \Phi$ 为 0.469 , 加入甲醛之后, 最大 吸收峰变为 $415 \mathrm{~nm}$, 最大发射波长为 $520 \mathrm{~nm}$, 均发生蓝 移, $\Phi$ 为 0.74 . 荧光强度与甲醛浓度 $(0 \sim 0.5 \mathrm{mmol} / \mathrm{L})$ 之 间存在很好的线性关系, 检测限低至 $5 \mu \mathrm{mol} / \mathrm{L}$. 经实验 证明，探针 6 可用于 $\mathrm{HeLa}$ 细胞中甲醛的成像，也可用于 活体斑马鱼(zebrafish)中甲醛分子的成像研究.

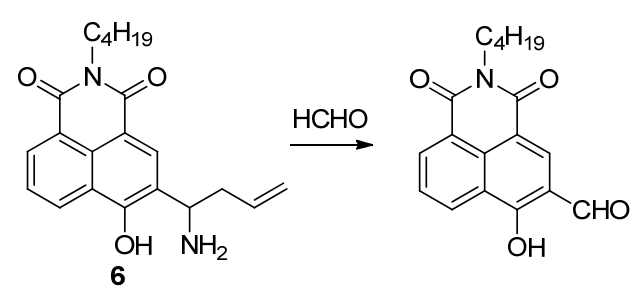

光动力治疗对于肿瘤的治疗具有准确性高、治疗范 围可以控制以及对人体产生的毒副作用较小等优势, 迅 速成为当前生物医学领域的研究方向. 但是, 我们知道 光的穿透力有限, 所以除了胃肠道这些可以利用内窥镜 进入的部位的癌症或皮肤癌等可以使用光疗技术，在其 他癌症的诊断和治疗方面, 光动力疗法的使用受到明显 的限制 ${ }^{[28]}$. 目前, 医学成像领域开发了一种常用的新技 术，即正电子发射断层扫描(PET)技术，这种技术主要 通过放射性核素产生正电子, 比较常见的就是放射性标 记的 ${ }^{18} \mathrm{~F}$-脱氧葡萄糖(FDG), 它是产生切伦科夫辐射的 理想光源. 葡萄糖所具有的结构与 FDG 相差不大, 于 是, FDG 在追踪能量代谢异常高的癌细胞中具有一定的 应用，在 PET 技术中 FDG 已经普遍用于肿瘤成像 ${ }^{[29]}$. Wilson 和 Chang 课题组 ${ }^{[30]}$ 设计了一个用于检测甲醛的 探针 7 (Eq. 3), 该探针与甲醛发生 Aza-cope 重排反应产 生放射性标记的 ${ }^{18} \mathrm{~F}$-脱氧葡萄糖(FDG), 从而可以实现 在活细胞以及生物体内检测甲醛分子, 这一工作为未来 在光动力治疗领域的研究和发展荧光探针提供了依据 和线索.

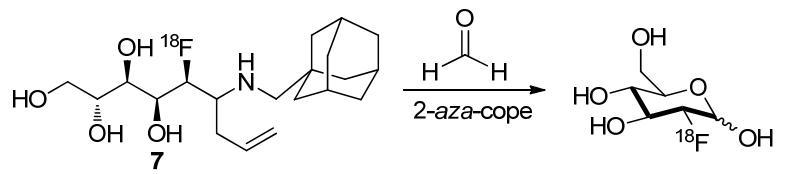

\section{2 基于探针分子中 $\mathrm{NHNH}_{2}$ 取代基与甲醛发生反应}

1,8-菜二甲酰亚胺衍生物分子中存在较大的共轭体 系，具有良好的光化学和热稳定性、高的苂光量子产率、 大的斯托克位移、化学可修饰性强、相对长的激发波长

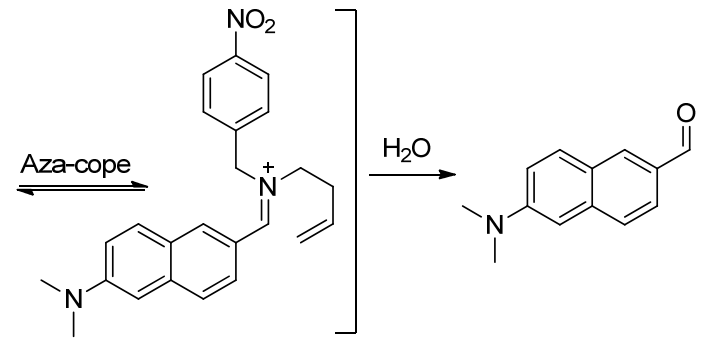

图式 5 探针 5 的结构及其对甲醛的响应机理

Scheme 5 Structure of the probe 5 and its responding reactions towards formaldehyde 
和发射波长以及双光子苂光等 ${ }^{[31]}$ 优点, 常被用于设计 苂光探针的母体. 2016 年, Lin 课题组 ${ }^{[32]}$ 以 1,8-荎二甲酰 亚胺为母体, $\mathrm{NHNH}_{2}$ 作为甲醛的反应位点, 设计出了一 种基于光诱导电子转移(PET)机理的荧光探针 8(如 Eq. 4 所示). 相对于 Aza-cope 重排反应, $\mathrm{NHNH}_{2}$ 作为甲醛的 反应位点具有快速检测甲醛并且克服了在生理条件下 反应达到平衡等缺点. 探针 $\mathbf{8}$ 在 PBS 缓冲溶液( 10 $\mathrm{mmol} / \mathrm{L}, \mathrm{pH}=7.4$, 含 $1 \%$ 的二甲亚砜)中, 加入甲酫, 在 $543 \mathrm{~nm}$ 处的苂光强度增加 325 倍, 检测限为 $7.1 \times 10^{-7}$ $\mathrm{mol} / \mathrm{L}$, 探针 8 与甲醛分子的反应速率为 $0.39 \mathrm{~min}^{-1}$. 相 对于其他生物体内的活性羰基化合物, 探针 8 对甲酫具 有较高的选择性. 探针 8 在 HeLa 细胞成像中几乎观察 不到苂光, 加入甲醛后, 可以看到明亮的细胞苂光成像. 除此之外, 还可以用于生物组织中内源性甲醛的成像研 究.

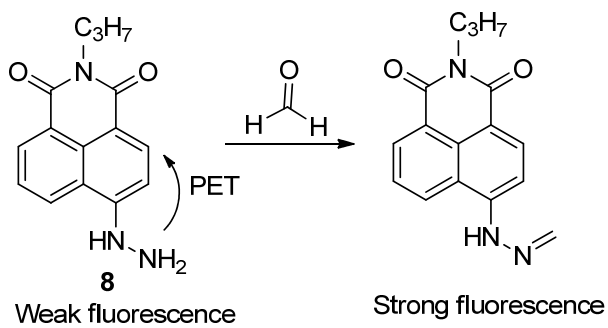

同年, 采用相同的识别机理, 通过改变 $\mathrm{N}$-上面的取 代基, Lin 和 Kin 等 ${ }^{[33]}$ 合成了以 1,8 -萗酰亚胺为苂光团, 生物素(Biotin)作为取代基的化合物 9 和氢原子为取代 基的化合物 $\mathbf{1 0}$ (如 Eq. 5 所示), 分别用于检测甲醛分子. 探针 9 连有生物素, 相对于探针 $\mathbf{1 0}$ 而言, 9 的生物相容 性好, 作者主要研究了探针 9 的性质. 在磷酸盐缓冲溶 液中, 加入甲醛后, 探针 9 在 $541 \mathrm{~nm}$ 处的苂光增强 140 倍. 在 $\mathrm{pH}$ 为 $4 \sim 7$ 的变化范围内, 探针 9 对甲酫分子具 有较高的灵敏度, 检测限为 $0.78 \mathrm{mmol} / \mathrm{L}$. 探针 9 在中性 或弱酸性环境的使用范围, 为其在癌细胞中检测甲醛提 供了可能. 除此之外, 探针 9 可以选择性的靶向定位于 生物素阳性受体(4T-1 细胞), 这项研究为探针 9 在肿瘤 部位跟踪内源性甲醛奠定了基础.

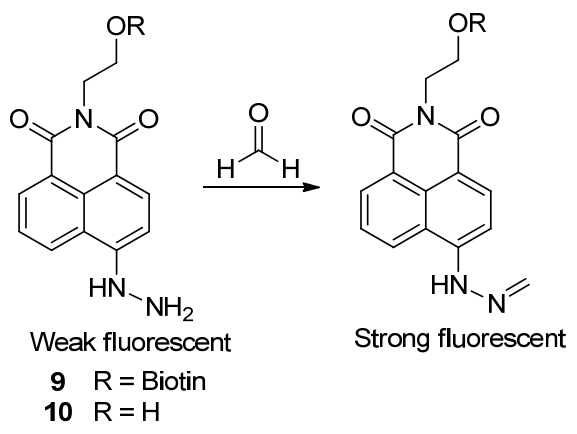

溶酶体作为一种细胞器, 主要参与细胞调亡、物质 代谢、细胞膜循环以及酶的形成等生命过程 ${ }^{[34]}$. 设计合 成能够定位某个特定细胞器的荧光探针近年来备受关 注 ${ }^{[35]}$. 吗啉基团的 $\mathrm{p} K_{\mathrm{a}}$ 值大约为 $5 \sim 6$, 溶酶体的 $\mathrm{pH}$ 范 围为 $4 \sim 5$, 吗啉质子化之后常存在于溶酶体中, 因此, 吗啉用于溶酶体的定位基团被广泛应用 ${ }^{[36]}$. 同样地, 2016 年, Lin 等 ${ }^{\left[{ }^{[7]}\right.}$ 也报道了以 1,8 -芸二甲酰亚胺为母体, $\mathrm{NHNH}_{2}$ 作为甲醛的反应位点, 并连接常规的吗啉基团 作为溶酶体的定位基团的荧光探针 11. 探针 11 与甲醛 的反应机理如 Eq. 6 所示, $\mathrm{NH}_{2}$ 取代基与甲醛发生反应 后, 形成 $\mathrm{C}=\mathrm{N}$ 双键, 给电子能力降低, PET 过程被抑制, 从而荧光恢复. 加入甲醛后, 在 PBS 缓冲溶液 (10 $\mathrm{mmol} / \mathrm{L}, \mathrm{pH}=7.4$, 含 $1 \%$ 的二甲亚砜)中, $543 \mathrm{~nm}$ 处的苂 光增强大约 350 倍, 检测限为 $5.02 \times 10^{-6} \mathrm{~mol} / \mathrm{L}$. 探针 $\mathbf{1 1}$ 对于甲醛的检测具有较高的选择性，对于其他的物质， 如乙二醛、甲基乙二醛、对羟基苯甲醛等活性羰基物质 均不会产生干扰. 探针 $\mathbf{1 1}$ 对甲醛的反应速率为 $0.37 \mathrm{~min}$ ${ }^{-1}$, 适用于生物系统的实时监测. 探针 11 主要集中在溶 酶体中, 并实现了 HeLa 细胞中甲酫分子的检测.

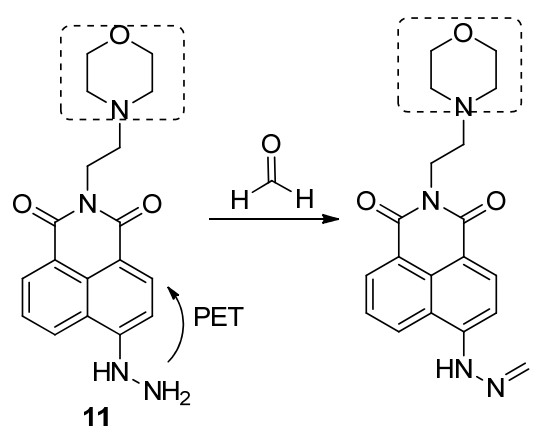

采用相同的识别机理, $\mathrm{Li}$ 和 $\mathrm{Yu}$ 课题组 ${ }^{[38]}$ 设计了由 8-羟基喹啉和苯并噻唑通过共轭组成的苂光探针 12 (如 图 12 所示). 探针 $\mathbf{1 2}$ 在水溶液中发出很微弱的荧光, 加 入甲醛后，在 $467 \mathrm{~nm}$ 处的苂光增强 5.5 倍，检测限低至 $900 \mathrm{nmol} / \mathrm{L}$. 作者通过理论计算进一步验证了探针 12 对 甲醛分子的响应变化机制.
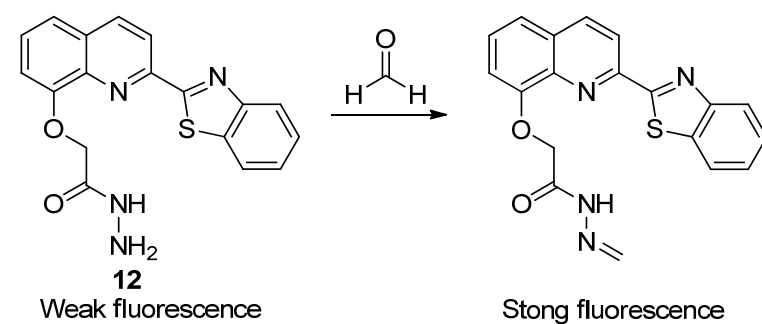

\section{3 基于探针分子中 $\mathrm{NH}_{2}$ 取代基与甲醛发生的反应}

Yoon 等 ${ }^{[39]}$ 报道了以 BODIY 为荧光团的苂光探针 13 (如Eq. 8 所示), 探针 13 与甲醛反应之前, 由于分子内 存在 PET 过程, 使探针分子中的荧光团的荧光发生猝 
灭, 加入甲醛之后, $\mathrm{NH}_{2}$ 取代基与甲醛发生反应, 形成 $\mathrm{C}=\mathrm{N}$ 双键, 给电子能力降低, 使 PET过程被中断, 从而 目标产物在 $\mathrm{pH}$ 为 8 的甲酸溶液中, 于 $535 \mathrm{~nm}$ 处的荧光 增强, 探针 13 对甲醛的检测限为 $165 \mathrm{nmol} / \mathrm{L}$. 这是一种 首次报道的基于 BODIY 为荧光团在中性环境中对于甲 醛分子的检测属于 “Turn-on” 型响应的苂光探针.

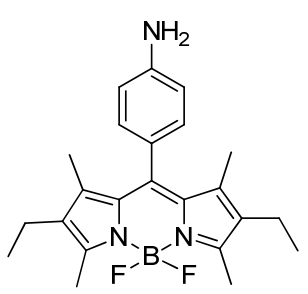

13

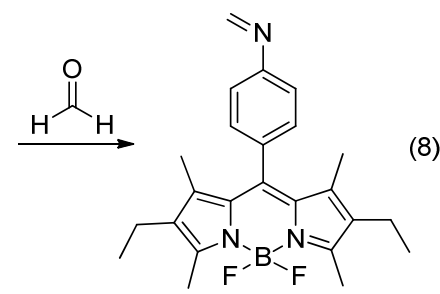

2016 年, Lin 课题组 ${ }^{[40]}$ 采用相同的识别机理, 基于 1,8-䒬酰亚胺为母体合成了用来识别甲醛的探针 $\mathbf{1 4}$ (如 Eq. 9 所示), 作者通过改变母体分子中 4 位上的取代基, 探究取代基的变化对探针分子的光谱性质的影响，当 1,8-䒬酰亚胺 4 位上被 $\mathrm{NH}$ 取代时, 分子内存在光诱导电 子转移(PET), 从而使得探针 14 在乙腈(含 $5 \%$ 的 HOAc) 溶液中, 表现出较弱的苂光, $\Phi$ 为 0.0073 , 加入甲醛, 4 $\min$ 之后, $512 \mathrm{~nm}$ 处的荧光强度显著增加, 与其他识别 甲醛的探针相比较而言, 探针 14 具有响应快速等特点. 探针 14 的合成与设计为后期甲醛类探针分子的进一步 发展打下了很好的基础.<smiles>NN1C(=O)c2cccc3c(N4CCNCC4)ccc(c23)C1=O</smiles>

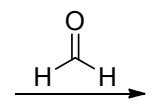

$\mathrm{H}$

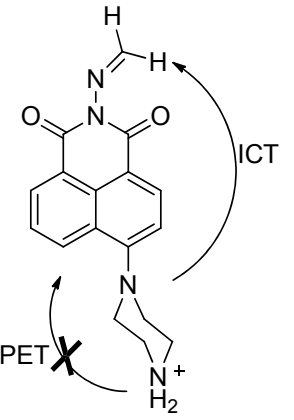

(9)

2016 年，与探针 14 的结构以及识别机理类似, Sun 课题组 ${ }^{[41]}$ 采用 1,8 -䒺酰亚胺为苂光团, 连接 $\mathrm{NH}_{2}$ 作为甲 醛的识别基团, 合成了探针 $\mathbf{1 5}$ (如 Eq. 10 所示). 作者探 讨了 1,8 -萗酰亚胺 4 位上的取代基, 发现化合物 15 相对 于其他化合物来说, 对甲醛具有较好的响应. 探针 15 对 甲醛的检测能实现肉眼识别, 并表现出较好的选择性, 检测限低至 $0.104 \mu \mathrm{mol} / \mathrm{L}$.

Thammakhet 等 ${ }^{[42]}$ 基于 5 -氨基荧光素为荧光基团, 连接聚乙烯醇长链, 利用 $\mathrm{NH}_{2}$ 与甲醛发生反应, 设计合 成了一种探针 16(如 Eq. 11 所示). 相对于其它用于检测 甲醛的探针而言, 探针 16 具有合成简单, 原料便宜易

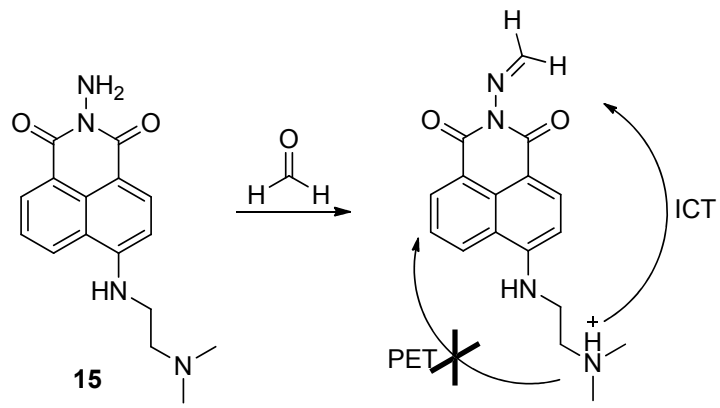<smiles>Nc1ccc(-c2c3ccc(=O)cc-3oc3cc([O-])ccc23)c(C(=O)[O-])c1</smiles>

16<smiles>C=Nc1ccc(-c2c3ccc(=O)cc-3oc3cc([O-])ccc23)c(C(=O)[O-])c1</smiles>

得, 并且连接聚乙烯醇长链使其具有一定的水溶性, 操 作简便等优点. 探针 16 苂光强度在甲醛浓度范围为 $5.0 \sim 100.0 \mu \mathrm{g} \cdot \mathrm{L}^{-1}$ 时, 表现出良好的线性关系, 检测限 为 $(3.820 \pm 0.079) \mu \mathrm{g} \cdot \mathrm{L}^{-1}$, 除此之外, 探针 $\mathbf{1 6}$ 对甲醛的 检测表现出较好的重现性 $(\mathrm{RSD}<2.5 \%)$. 探针 16 能用于 蔬菜、水果以及海鲜中甲醛含量的检测, 还不能用于活 细胞中甲醛的检测.

Lin 课题组 ${ }^{[43]}$ 以罗丹明 $6 \mathrm{G}$ 为荧光团, 采用两步反 应合成了化合物 17, 如 Scheme 6 所示. 探针 17 分子内 存在一个封闭的五元环, 表现出较弱的苂光, 与甲醛发 生反应后，五元环被破坏，目标产物的荧光恢复，从而 实现对甲醛分子的识别. 在磷酸盐缓冲溶液 $(25 \mathrm{mmol} / \mathrm{L}$, $\mathrm{pH}=7.4$, 含 $50 \%$ 的二甲亚砜)中, 探针 17 的苂光量子产 率为 0.21 , 加入一倍甲醛分子后, 在 $560 \mathrm{~nm}$ 处的苂光增 强 7.4 倍, 溶液颜色发生变化(无色变为粉色), 探针 $\mathbf{1 7}$ 对甲醛分子具有较好的选择性和灵敏度, 检测限为 $7.7 \times 10^{-7} \mathrm{~mol} / \mathrm{L}$. 像大多数探针一样, 探针 17 可以用于 HeLa 活细胞中甲醛的成像研究, 也可用于像干香菇等 食物中甲醛的识别, 并且是首个能实现对甲醛气体的裸 眼识别的荧光探针, 为生物系统、食品工业以及生活环 境中甲醛分子的检测提供了指导.

\section{4 金属配合物用于检测甲醛的荧光探针}

2014 年, $\mathrm{Li}$ 等 ${ }^{[44]}$ 基于金属镝(Dy)和 2-氨基-1,4-苯二 甲酸 $\left(\mathrm{H}_{2} \mathrm{aBDC}\right)$ 合成了大小可控的有机金属配位聚合物 $\left(\mathrm{Dy}_{2} \mathrm{aBDC}_{3}\right) . \mathrm{Dy}_{2} \mathrm{aBDC}_{3}$ 聚合物粒子无论是在固态还是 分散在 DMF，乙醇，丙酮等溶剂中都没有苂光，但是， 当有少量的水存在时，聚合物显示出较强的荧光; 当聚 合物分散在含有醛类化合物像甲醛、乙醛和丙醛的 DMF 溶液中时, 在 $365 \mathrm{~nm}$ 波长的紫外灯下照射, 可观测到荧 光强度增强，随着醛类化合物的含量的增高，苂光强度 


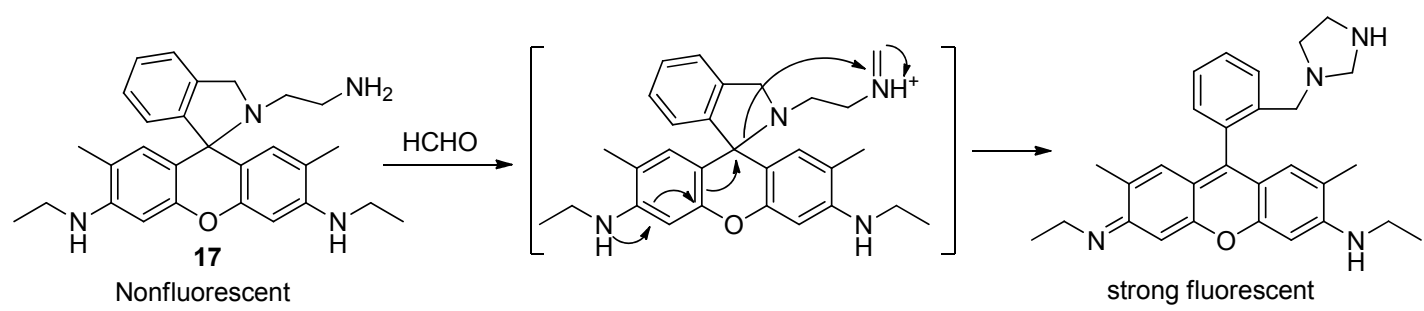

图式 6 探针 17 的结构及其对甲醛的响应机理

Scheme 6 Proposed illuminating fluorescent formaldehyde probe 17

不断增强并且发生不同程度的红移. 从诱导发生红移的 程度相比较而言, $\mathrm{Dy}_{2} \mathrm{aBDC}_{3}$ 聚合物粒子对于甲醛的灵 敏度要高于乙醛和丙醛.

Fang 课题组 ${ }^{[45]}$ 通过 “点击化学” 设计合成了化合 物 18 (Eq. 12 所示), 分子中杯 [4]芳烃为母体, 1,2,3-三唑 为连接基团和识别基团, 双-硝基苯并噁二唑(NBD)为 荧光发射基团. 在化合物 $\mathbf{1 8}$ 的 THF 溶液中对 $\mathrm{Ag}^{+}$的识 别具有较高的选择性, 不断改变加入 $\mathrm{Ag}^{+}$的浓度, 可以 发现, 化合物在 $527 \mathrm{~nm}$ 处的发射峰不断降低, 并且在 $576 \mathrm{~nm}$ 处出现一个新的吸收峰. 化合物 18 对 $\mathrm{Ag}^{+}$的检 测限为 $6.2 \times 10^{-7} \mathrm{~mol} / \mathrm{L}$. 考虑到甲醛是一种强还原剂, 能快速的把 $\mathrm{Ag}$ 配合物中的配位中心 $\mathrm{Ag}^{+}$还原为 $\mathrm{Ag}^{0}$. 作 者向 18- $\mathrm{Ag}^{+}$混合溶液中不断改变加入甲醛的浓度, 可 以发现, 在 $576 \mathrm{~nm}$ 处的苂光吸收峰不断降低, 在 527 $\mathrm{nm}$ 处出现新的吸收峰. 18- $\mathrm{Ag}^{+}$混合溶液对 $\mathrm{HCHO}$ 的识 别的检测限低至 $1 \mu \mathrm{mol} / \mathrm{L}$. 这一配位和还原过程可以被

\section{重复至少五次左右.}

\section{5 其他机理检测甲醛}

2015 年, Wong 等 ${ }^{[46]}$ 主要通过 $\mathrm{Au}(\mathrm{III})$ 化合物催化三 组分偶合反应 $\left(\mathrm{A}^{3}\right.$-coupling reaction)设计了一种新型的 快速检测 $\mathrm{HCHO}$ 的方法. 这种检测方法的机理为连接 树脂的胺、甲醛以及连有苂光团的端炔在 $\mathrm{Au}(\mathrm{III})$ 催化作 用下发生三组分偶合反应，生成具有荧光的目标化合 物, 从而达到对 HCHO 的识别, 机理如 Eq. 13 所示.

Liu 课题组 ${ }^{[47]}$ 利用 $\mathrm{HCHO}$ 能将 $\mathrm{Ag}^{+}$还原成 $\mathrm{Ag}$, 设计 了 $20 \sim 30 \mathrm{~nm}$ 的 $\mathrm{Au}$ 核-银壳纳米离子 $(\mathrm{Au} @ \mathrm{AgNRs})$, $\mathrm{Au} @ A g N R s$ 通过苂光光谱法和比色法选择性地检测甲 醛分子. 在 AuNRs- $\mathrm{Ag}^{+}$-甘氨酸-氢氧化钠缓冲溶液中, AuNRs 在 748 和 $555 \mathrm{~nm}$ 处出现吸收带, 利用 $\mathrm{HCHO}$ 将 $\mathrm{Ag}^{+}$还原为 $\mathrm{Ag}^{0}$ 包裹在 $\mathrm{AuNRs}$ 的表面上以形成 $\mathrm{Au} @ \mathrm{Ag}$ 沉淀, 随着甲醛的加入, $748 \mathrm{~nm}$ 处的吸收带不断发生红
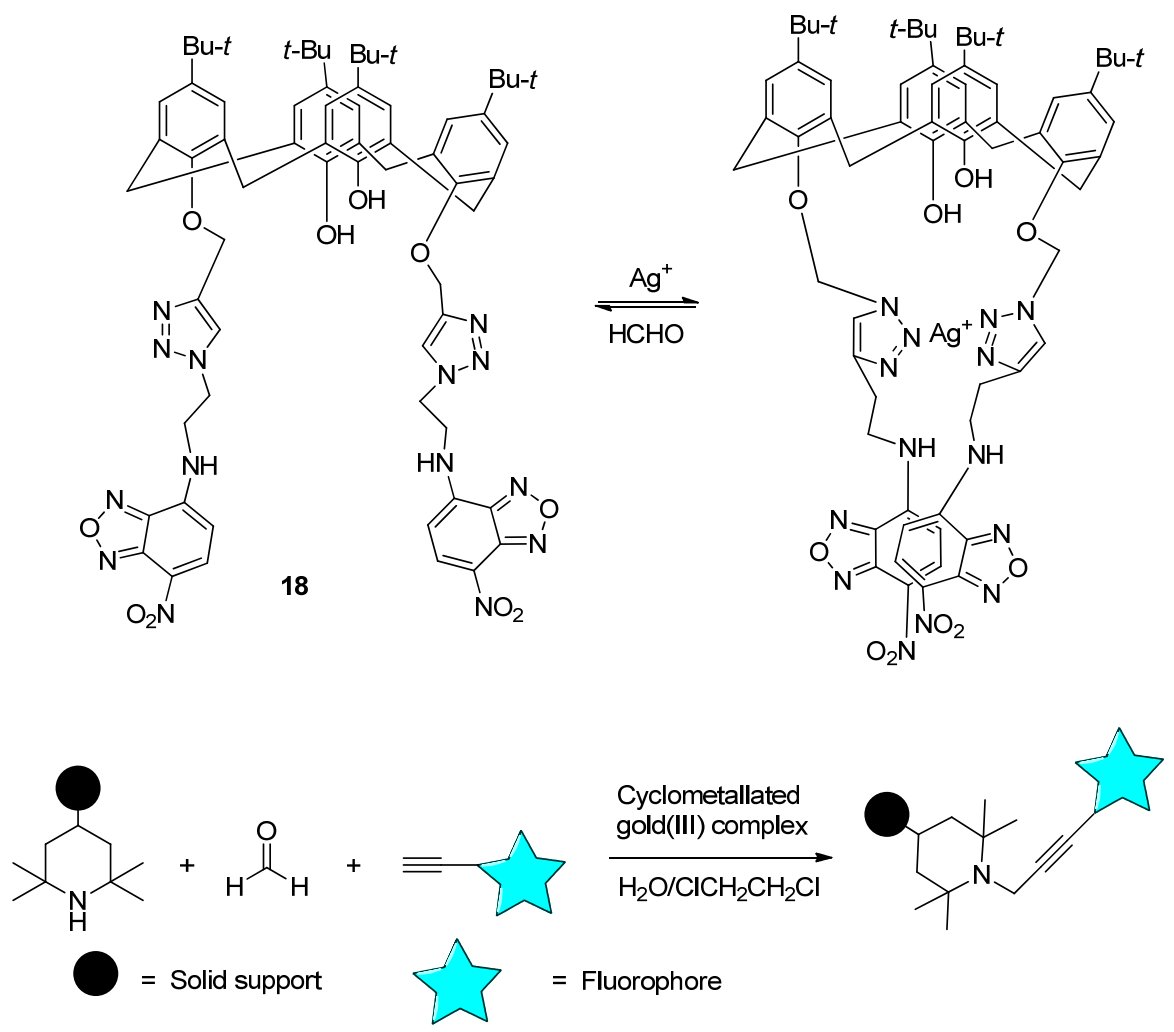
移, 并且溶液颜色也在不断发生变化. $\mathrm{Au}$ 核- $\mathrm{Ag}$ 壳纳米 离子( $\mathrm{Au} @ \mathrm{AgNRs})$ 对甲醛的检测具有较高的选择性, 检 测限低至 $6.3 \times 10^{-11} \mathrm{~g} / \mathrm{mL}$. Au@AgNRs 纳米粒子广泛应 用于水样中甲醛的检测, 并且展现出广泛的实用性.

\section{2 检测甲基乙二醛的荧光探针}

甲基乙二醛作为生物体内活性羰基化合物的一种, 伴随着脂质过氧化、非酶糖基化等能量代谢过程而产 生 ${ }^{[48]}$. 甲基乙二醛有较高的反应活性, 易与生物大分子 如 DNA, 蛋白质等物质的氨基和颈基发生反应, 生成糖 基化终产物(ACEs) ${ }^{[49]}$. 研究表明, 人体内的糖尿病、心 血管疾病 ${ }^{[50]}$ 、痛觉过敏 ${ }^{[51]}$ 以及肾脏疾病 ${ }^{[52]}$ 均与甲基乙二 酫含量的升高有较大的关系. 近年来, 科研工作者对甲 基乙二醛的研究已经有了许多进展, 但甲基乙二醛在细 胞过程中的作用和发病机制仍然很少被了解 ${ }^{[4 a]}$. 因此, 发展和研究用于生物体系中甲基乙二醛含量的检测和 成像成为当前研究的热点. 目前, 关于甲基乙二醛荧光 探针的报道主要是基于邻苯二甲胺识别基团与甲基乙 二醛发生的反应.

2013 年, Spiegel 等 ${ }^{[53]}$ 首次报道了 3 种基于 BODIY 为母体, 连接邻苯二甲胺作为识别基团, 通过改变 BODIY 和邻苯二甲胺上面的取代基用于检测甲基乙二 醛的荧光探针 19 21, 如 Eq. 14 所示. 探针 19 21 的 识别机理主要是由于探针分子内的邻苯二甲胺基团与 BODIY 基团之间存在 a-PET 过程, 当邻苯二甲胺基团 与甲基乙二醛反应后, 这一过程被抑制, 从而使目标化 合物的荧光恢复. 作者通过理论计算预测到探针 19 与 甲基乙二醛反应后发生强烈的苂光增强, 探针 $\mathbf{2 0}$ 其次, 探针 21 产生较小的变化. 实验表明, 探针 19 与甲基乙
二醛反应后, 苂光强度增加 10 倍, $\Phi$ 由 0.003 增加为 0.326 , 而探针 20 仅发生中等强度的荧光增强，对于探 针 21 而言, 响应很小. 于是, 作者主要对探针 19 做了进 一步研究. 在 PBS 缓冲溶液中，探针 19 与甲基乙二醛共 存 $1 \mathrm{~h}$ 的检测限为 $50 \sim 100 \mathrm{nmol} / \mathrm{L}$, 并且共存的时间越 长, 检测限也越高. 探针 19 与甲基乙二醛的一阶速率常 数为 $4.56 \times 10^{-3} \mathrm{~s}^{-1}$. 探针 $\mathbf{1 9}$ 不仅适用于 HeLa 活细胞中 甲基乙二醛分子的成像研究，同时，也能基于高效液相 色谱法应用于血清或血浆中甲基乙二醛浓度的检测，为 后期各种疾病的诊断提供了便利.<smiles></smiles><smiles></smiles>

$19 \mathrm{R}^{1}=$ COOEt, $\mathrm{R}^{2}=\mathrm{CH}_{3}$

$20 \mathrm{R}^{1}=$ COOEt, $\mathrm{R}^{2}=\mathrm{H}$

$21 R^{1}, R^{2}=H$

三苯基膦正离子带正电荷，线粒体膜外带负电荷， 因此三苯基膦取代基作为线粒体的定位基团，常被用于 设计合成某些生物小分子的苂光探针 ${ }^{[54]}$. Murphy 等 ${ }^{[5]}$ 采用邻苯二甲胺作为甲基乙二醛和乙二醛的反应位点, 连接三苯基膦定位基团合成了探针 22(如 Scheme 7 所 示). 作者发现，化合物 22 对甲基乙二醛的反应活性和 灵敏度要高于乙二醛，这与甲基乙二醛对生物体的毒性 要强于乙二醛的说法一致. 除此之外，作者通过探针 22 研究了细胞体内甲基乙二醛与乙二酫的变化与线粒体

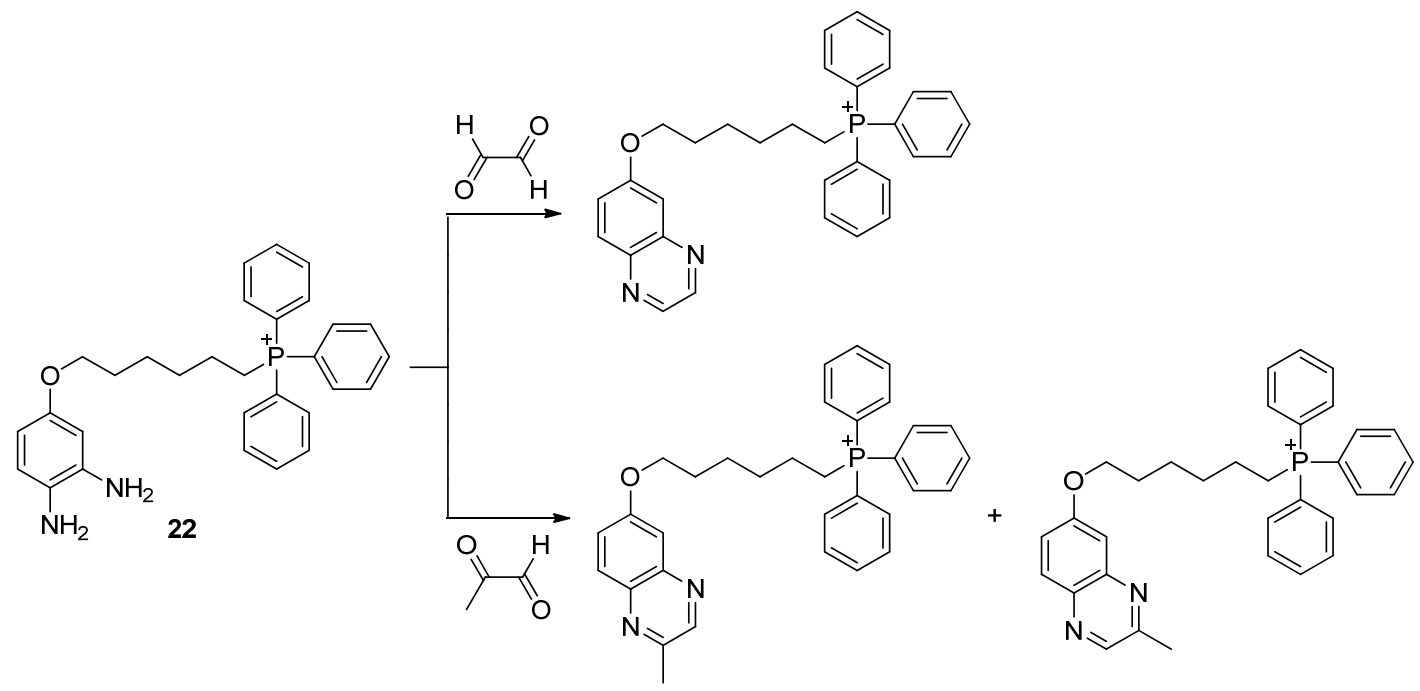

图式 7 探针 22 与甲基乙二醛和乙二醛的反应产物

Scheme 7 Reaction of $\mathbf{2 2}$ with methyl glyoxal or glyoxal to form quinoxaline products 
指出了方向.

基于上述相同的识别机理, 2015 年, Zhou 课题组 ${ }^{[56]}$ 采用 4-溴-1,8-萗二甲酸酐为原料, 首次设计合成了一个 用于检测甲基乙二醛的双光子苂光探针 23(如 Eq. 15 所 示). 在 PBS 缓冲溶液 (10 mmol/L, $\mathrm{pH}=7.4$, 含 $10 \%$ 的二 甲亚砜)中, 探针 23 几乎没有荧光, 加入甲基乙二醛后, 在 $528 \mathrm{~nm}$ 处苂光增强 33 倍, $\Phi$ 由 0.029 变为 0.256 . 在 甲基乙二醛浓度范围为 $0 \sim 10 \mu \mathrm{mol} / \mathrm{L}$ 范围内, 探针 23 的苂光强度与甲基乙二醛的浓度呈现出良好的线性关 系. 探针 23 对甲基乙二醛的检测具有较高的选择性和 灵敏性, 检测限为 $77 \mathrm{nmol} / \mathrm{L}$. 除此之外, 探针 23 可以 用于 HeLa 活细胞中甲基乙二醛分子检测的双光子成像 研究.
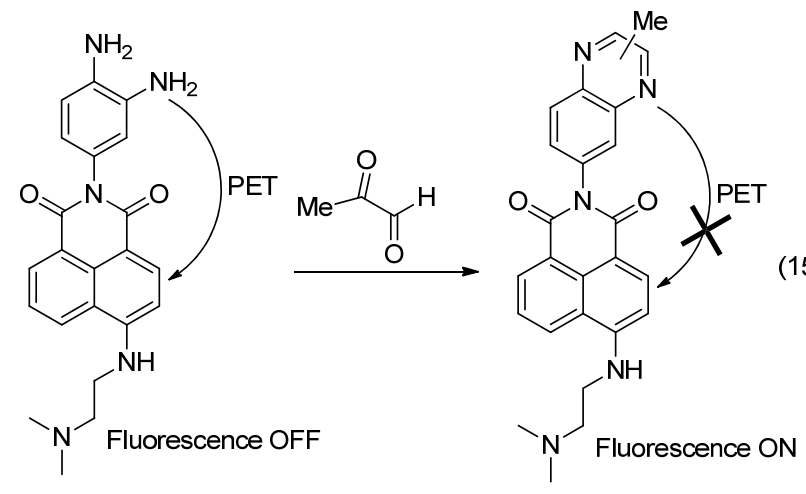

23

超分子水凝胶具有生物相容性和可降解性等 ${ }^{[57]}$ 优 势, 被广泛用于伤口愈合 ${ }^{[58]}$, 药物释放 ${ }^{[59]}$ 以及组织工程 等 ${ }^{[60]}$ 领域, 近年来, Liang 及其课题组 ${ }^{[61]}$ 设计合成了基 于联吡啶的超分子凝胶用于可以检测 $\mathrm{Cd}^{2+}$, 并且伴随 着选择性好和灵敏度高的特征, 为这一技术在分析检测 领域的应用提供了基础. 类似地, 2016 年, Liang 等 ${ }^{[62]}$ 基
于邻苯二甲胺与甲基乙二醛具有较高的反应活性, 设计 合成了一种水凝胶前体 $\mathbf{2 4}$ (如 Eq. 16 所示), 可以原位点 击(in situ “click”)与甲基乙二醛发生反应形成化合物 $\mathbf{2 5}$, 然后化合物 25 自组装形成超分子水凝胶, 从而达到原 位检测和可视化识别，并且作者设计合成的化合物 24 对甲基乙二醛的识别不受 NO 的干扰, 具有较高的选择 性

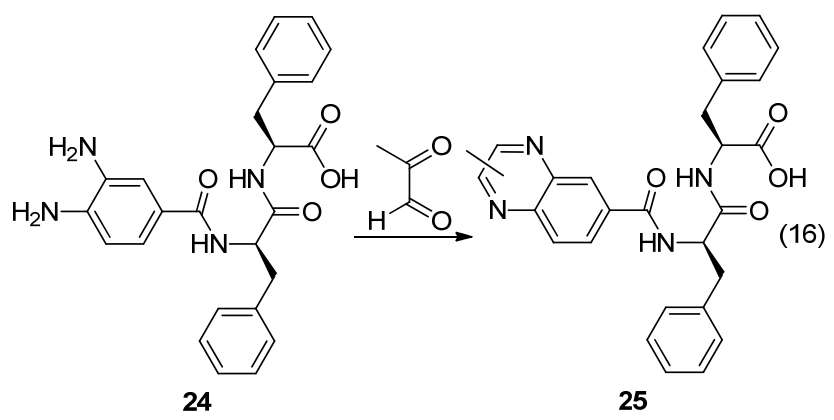

Kool 课题组 ${ }^{[63]}$ 基于苂光腙设计合成了探针 26 (如 Eq. 17 所示), 用于甲基乙二醛的标记和检测. 苂光腙分 子的设计需满足以下几个条件：在环境条件下，对水具 有较高的稳定性，不易发生水解; 荧光发生完全猝灭, 几乎观察不到背景的苂光; 必须是热力学不稳定的腙, 从而能驱使反应的发生. 探针 26 分子设计中 2-肼基吡 啶作为亲核试剂, 实现对甲基乙二醛分子的识别, 偶氮 苯作为苂光猝灭基团, 5-甲氧基邻氨基苯甲酸作为催化 剂. 在磷酸盐缓冲溶液中，探针 26 与甲基乙二醛反应 后, 苂光强度增强 30 倍. 作者通过催化剂的选择, 底物 范围以及醛的结构等方面对这一反应进程的影响进行 了探讨. 探针 26 适用于活细胞中甲基乙二醛分子的成 像研究.

2017 年, Zeng 等 ${ }^{[64]}$ 基于邻二氨基罗丹明母体设计 合成了探针 27(如 Scheme 8 所示), 可以实现对甲醛 “Turn-ON” 型以及对甲基乙二醛 “Turn-OFF” 型的检测.

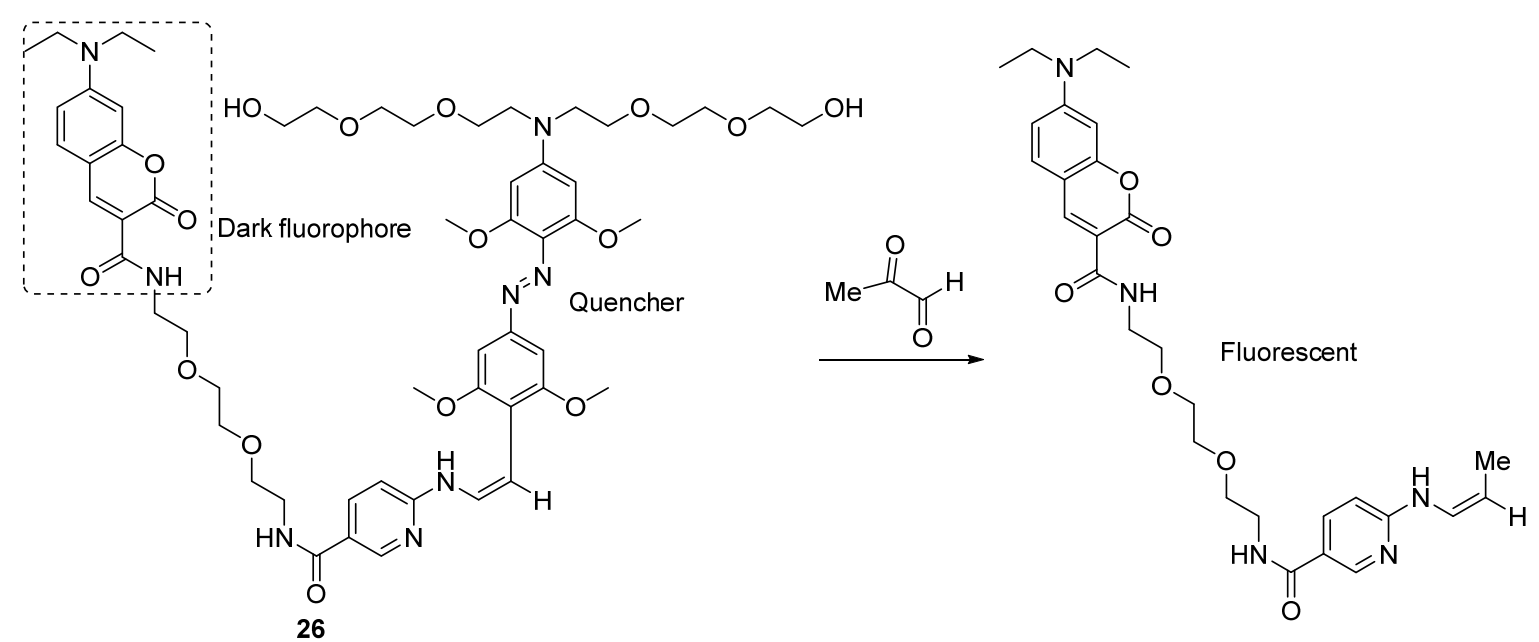




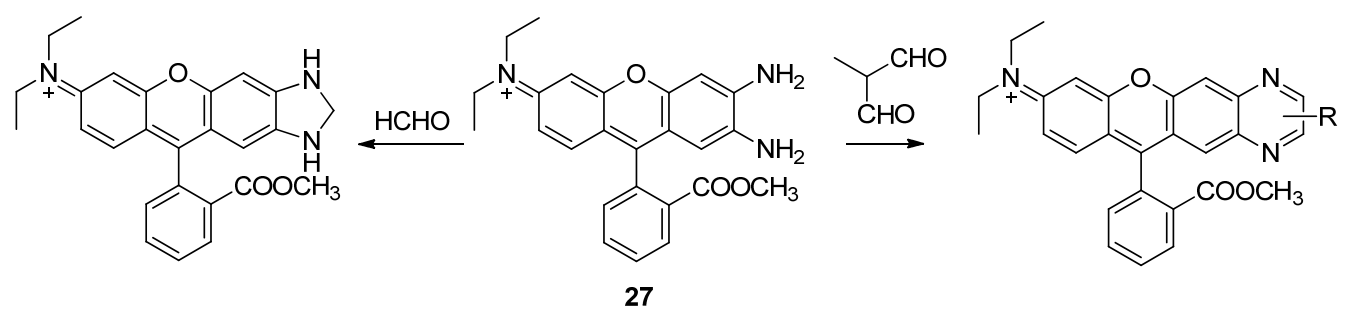

图 8 探针 $\mathbf{2 7}$ 的结构及其对甲醛及甲基乙二醛的反应产物

Figure 8 Structure of the probe 27 and its proposed reaction products with formaldehyde and methylglyoxal

在 Tris- $\mathrm{HCl}$ 缓冲溶液 $(10 \mathrm{mmol} / \mathrm{L}, \mathrm{pH}=7.4$, 含 $30 \%$ 的乙 醇)中, 探针 27 在 $642 \mathrm{~nm}$, 处几乎没有苂光, 加入甲醛 后, 发射波长蓝移至 $620 \mathrm{~nm}, \Phi$ 由 0.07 增加为 0.55 , 探 针 27 对甲醛分子的检测限为 $8.3 \mu \mathrm{mol} / \mathrm{L}$. 加入甲基乙二 醛之后, 探针 27 的发射波长红移至 $642 \mathrm{~nm}$, 苂光强度 降低, $\Phi$ 由 0.07 降低为 0.0014 . 同样地, 探针 27 也能用 于活细胞中实现对甲醛 “Turn-ON” 型以及对甲基乙二 醛 “Turn-OFF” 型的成像研究.

钓和铱的配合物因具有较好的光物理性质和电化 学性质, 常被用来作为生物小分子以及离子的探针 ${ }^{[6]}$. 2017 年, Zhang 课题组 ${ }^{[66]}$ 基于邻苯二甲胺取代的菲咯啉 和联吡啶报道了一种钓的配合物 28 和一种铱的配合物 29, 用于对甲基乙二酫分子的检测, 如 Eq. 18 所示. 由 于探针分子内存在光诱导电子转移(PET)过程, 使其在 磷酸盐缓冲溶液中表现出较弱的荧光, 探针 $\mathbf{2 8}$ 和 $\mathbf{2 9}$ 的 荧光量子产率分别为 $0.12 \%$ 和 $0.23 \%$; 与甲基乙二醛反 应后, PET 过程被抑制, 探针在 $\mathrm{pH} 3 \sim 11$ 范围内, 表现 出强烈的荧光. 化合物 $\mathbf{2 8}$ 和 $\mathbf{2 9}$ 对甲基乙二醛的检测均 具有一定的选择性, 探针 28 的检测限为 $0.78 \mu \mathrm{mol} / \mathrm{L}$, 化合物 29 为 $1.15 \mu \mathrm{mol} / \mathrm{L}$. 作者通过密度泛函理论对这 一识别机理进行了计算. 除此之外, 探针 28 和 29 具有 较好的生物相容性, 可以成功地用于 RAW 264.7 巨噬细 胞和 D. magna 中的成像研究.

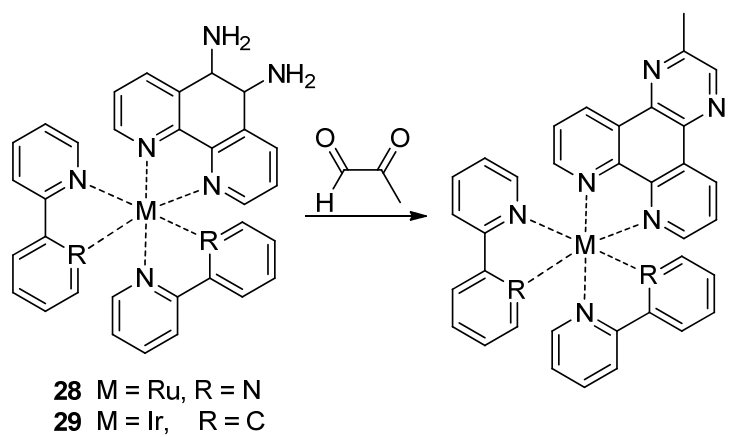

(18)

\section{3 检测丙二醛的荧光探针}

氧化应激(Oxidative Stress)作为生命体内的一种非 健康状态, 主要是通过生命体内氧化过程及抗氧化系统
失衡所引起, 这一状态与多种疾病, 如心血管疾病、肿 瘤及糖尿病等的产生和发展有着不可分割的联系 ${ }^{[67]}$. 丙二醛(Malondialdehyde, MDA)作为生物体内活性羰基 化合物的一种类型, 是氧化应激的一种经典的生物标志 物 ${ }^{[68]}$, 生物体内氧化应激的失衡程度可以通过机体内 丙二醛含量的高低体现出来 ${ }^{[69]}$, 于是, 丙二醛也可以作 为一种重要的病理指标, 当生物体内, 丙二醛含量的升 高会引起一序列的疾病, 如白血病、糖尿病、癌症、心 血管疾病、哮喘以及动脉弹样硬化肝脏疾病等 ${ }^{[00]}$.

2015 年, Liu 课题组 ${ }^{[71]}$ 以可与 MDA 作用的苯甲酰肼 为起始物, 通过对反应机理的深入研究构建了可用于体 内成像的苂光探针 30 (Scheme 9), 首次实现了 MDA 在 活细胞内的成像. Gleiter 课题组 ${ }^{[72]}$ 报道 2,4-二硝基苯甲 酰胺由于取代基硝基具有强的吸电子效应，使得其与 MDA 具有较高的反应活性, 于是, 作者通过改变苯甲 酰肼上取代基的位置和类别, 探索出了与 MDA 反应活 性较高的苯甲酰肼, 作为 MDA 的识别基团, 然后通过 $\mathrm{NH}$ 基团将 1,8-萗酰亚胺荧光基团连接起来, 设计合成

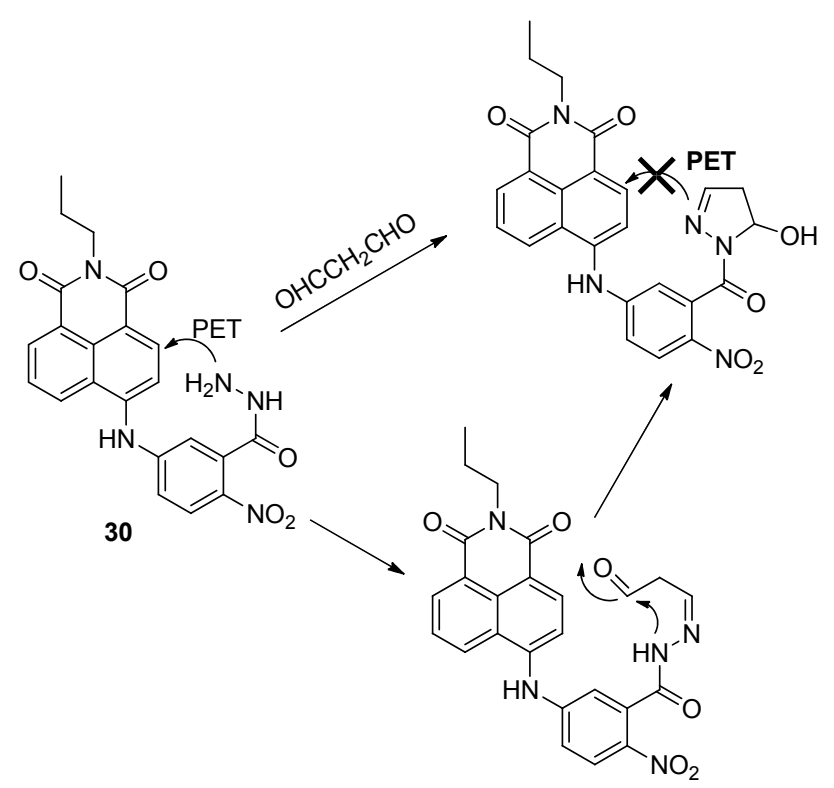

图式 9 探针 30 的结构及其对丙二醛的反应机理 Scheme 9 Structure of $\mathbf{3 0}$ and its reaction mechanism of malondialdehyde 
了探针 30. 探针 30 分子内存在 PET 过程, 在 DMSO磷酸盐缓冲溶液 $(V: V=1: 9,50 \mathrm{mmol} / \mathrm{L}, \mathrm{pH}=7.4)$ 中, 探针 30 表现出较弱的苂光, 与 MDA 发生反应后, PET 过程被抑制, 在 553 和 $520 \mathrm{~nm}$ 处荧光分别增强 174 倍和 52 倍. 探针 30 对 MDA 的识别具有较高的选择性, 检测 限低至 $0.6 \mu \mathrm{mol} / \mathrm{L}$. 探针 30 首次实现了 $\mathrm{MDA}$ 在活细胞 内的成像研究, 它不仅可以检测内源性的 MDA, 还可 以实时追踪肿溜细胞的内源性 MDA 及氧化应激状态下 MDA 的发生. 这一工作促进了后期氧化应激相关病理 过程的探究及抗氧化药物的开发.

\section{4 检测丙烯醛的荧光探针}

丙烯醛是一种高毒性的不饱和醛，它可以通过油、 木炭、木材、塑料和其他有机物质的不完全燃烧而产生, 也可以通过多胺、脂质、氨基酸和其他生物分子的氧化 应激下内源产生 ${ }^{[73]}$. 丙烯醛具有高反应性, 常会引发许 多免疫疾病和炎症等 ${ }^{[74]}$, 被认为是对人类健康的真正 危害. 发展新型的、有效的用来检测丙烯醛的方法显得 至关重要.

2014 年, Nagano 等 ${ }^{[75]}$ 基于四甲基羧基罗丹明为母 体设计合成了荧光探针 31(如 Scheme 10 所示), 用于快
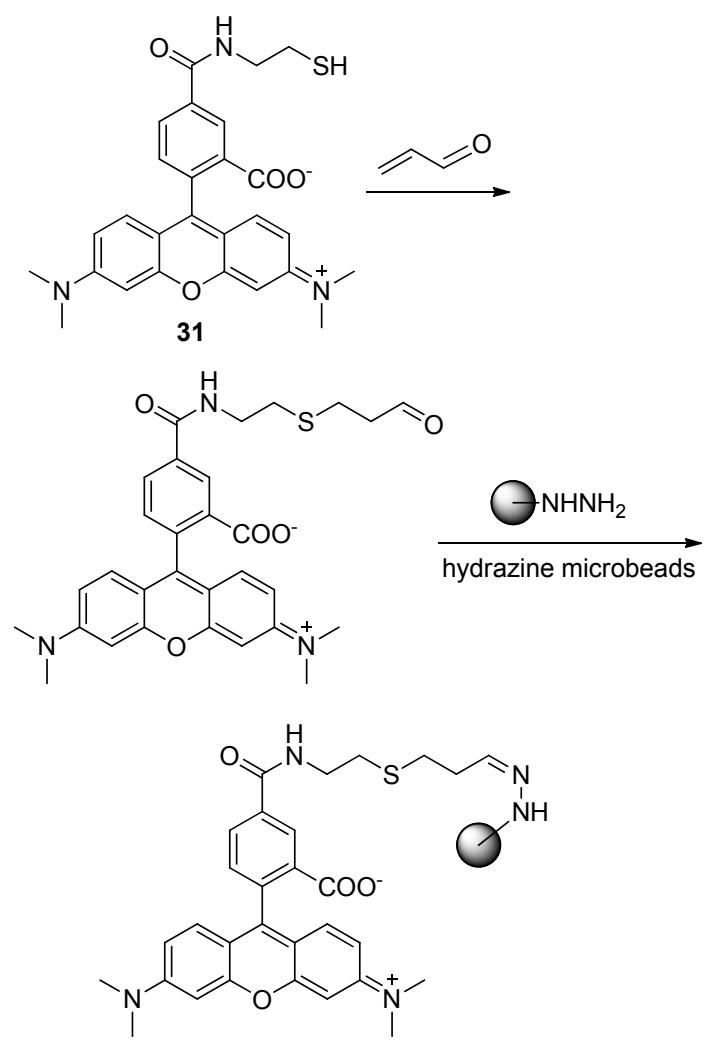

图式 10 探针 31 的结构及其与丙二醛的反应 Scheme 10 Structure of $\mathbf{3 1}$ and its strategy for detection of acrolein
速检测血浆里面的丙烯醛. 探针与丙烯醛的识别过程分 两步进行，首先探针 31 与含丙烯醛的血浆发生迈克尔 加成反应，然后加成产物与附有聚乙二醇且含有 $\mathrm{NHNH}_{2}$ 官能团的复合微球作用，从而通过共价键将探 针分子连接在复合微球上. 复合微球与含有丙烯醛的溶 液混合后，表现出较强的苂光，并且其他活性羰基化合 物产生的干扰小. 这种方法与传统的检测方法相比，具 有操作简单、便宜、实用、灵敏度高等优势，检测限为 $0.54 \mu \mathrm{mol} / \mathrm{L}$. 作者指出这一方法潜在的缺陷是探针 $\mathbf{3 1}$ 对丙烯醛的检测并不能从严格意义上与其他某些活性 羰基化合物区分开，但是这不能阻碍它在疾病诊断以及 临床上面的应用.

\section{5 总结与展望}

活性羰基化合物作为生物体内重要的活性物种之 一, 其在人体内的含量发生异常时, 会引发许多疾病, 设计合成用于快速、高效、实时监测羰基化合物含量的 探针在生理, 病理以及疾病诊断和控制方面的研究具有 重要的意义. 本文根据活性羰基化合物的种类，归纳和 总结了近年来用于检测甲醛、甲基乙二醛、丙二醛以及 丙烯醛等荧光探针的设计, 识别机理以及应用. 通过综 述发现，目前关于活性羰基化合物的研究主要集中在甲 醛和甲基乙二醛，这是因为目前发现了较多对甲醛或甲 基乙二醛物种的特异性有机反应，而其它活性羰基化合 物物种的特异性有机反应相对较少. 所以，今后应当寻 找某种新的用来特异性识别某种活性羰基化合物的有 机反应. 除此之外, 部分探针存在不能用于微生物环境, 选择性不好，灵敏性不高，发射未达到近红外区等不足， 今后设计新的用来识别活性羰基化合物的荧光探针可 以从以下几个方面进行优化：选择近红外发射的荧光 团; 与目标化合物结合之后显示 “Switch-ON” 型荧光 响应或者比率型荧光探针; 探针与活性羰基化合物选择 性识别之后, 产物的荧光强度要高, 苂光量子产率也应 该较好; 探针具有较低的细胞毒性和较好的生物相容 性, 最好是水溶性或两亲性的荧光探针; 探针与某种活 性羰基化合物的识别具有较高的灵敏性和选择性; 进一 步开发和设计出双光子, 近红外还能定位在某一细胞器 中的选择性荧光探针, 从而实现细胞器的功能成像. 还 有一点, 目前荧光探针的成像研究主要进行到细胞成像 阶段, 少部分进行到活体组织成像阶段. 今后设计出能 对生物体进行原位、实时、动态的可视化监测的高选择 性和灵敏度的活性羰基化合物荧光探针, 开拓其在疾病 诊断和临床方面的应用已成为主要研究方向, 也是当今 化学与生命科学研究的重点和难点. 


\section{Referenes}

[1] (a) Kalapos, M. P. Diabetes Res. Clin. Pract. 2013, 99, 260. (b) Onyango, A. N. Chem. Phys. Lipids 2012, 165, 777.

[2] Semchyshyn, H. M. Sci. World J. 2014, 2014, 10.

[3] (a) Colzani, M.; De Maddis, D.; Casali, G.; Carini, M.; Vistoli, G.; Aldini, G. ChemMedChem 2016, 11, 1778.

(b) Regazzoni, L.; De Courten, B.; Garzon, D.; Altomare, A.; Marinello, C.; Jakubova, M.; Vallova, S.; Krumpolec, P.; Carini, M.; Ukropec, J.; Ukropcova, B.; Aldini, G. Sci. Rep. 2016, 6, 27224.

(c) Hwang, S.; Lee, Y.-M.; Aldini, G.; Yeum, K.-J. Molecules 2016, 21,280 .

(d) Biswas, S.; Mano, J. I. Plant Cell Physiol. 2016, 57, 1432.

[4] (a) Nemet, I.; Varga-Defterdarović, L.; Turk, Z. Mol. Nutr. Food Res. 2006, 50, 1105 .

(b) Kato, S.; Burke, P. J.; Koch, T. H.; Bierbaum, V. M. Anal. Chem. 2001, 73, 2992.

(c) Soman, A.; Qiu, Y.; Chan Li, Q. J. Chromatogr. Sci. 2008, 46, 461 .

[5] (a) Jung, H. S.; Chen, X.; Kim, J. S.; Yoon, J. Chem. Soc. Rev. 2013, 42, 6019 .

(b) Niu, L.-Y.; Chen, Y.-Z.; Zheng, H.-R.; Wu, L.-Z.; Tung, C.-H.; Yang, Q.-Z. Chem. Soc. Rev. 2015, 44, 6143.

(c) Wang, S.; Shen, S.; Zhang, Y.; Dai, X.; Zhao, B. Chin. J. Org. Chem. 2014, 34, 1717 (in Chinese).

(王胜清, 申世立, 张延如, 戴溪, 赵宝祥, 有机化学, 2014, 34, 1717.)

[6] (a) Santos-Figueroa, L. E.; Moragues, M. E.; Climent, E.; Agostini, A.; Martinez-Manez, R.; Sancenon, F. Chem. Soc. Rev. 2013, 42, 3489 .

(b) Li, B.; Meng, W.; Yang, M.; Su, N.; Liu, X.; Yang, B. Chin. J. Org. Chem. 2015, 35, 2629 (in Chinese).

(李波，孟文斐，杨美盼，苏娜，刘晓静，杨秉勤，有机化学, 2015, 35, 2629.)

[7] (a) Guo, Z.; Park, S.; Yoon, J.; Shin, I. Chem. Soc. Rev. 2014, 43, 16.

(b) Wang, X.-D.; Wolfbeis, O. S. Chem. Soc. Rev. 2014, 43, 3666

(c) Zhang, R.; Zhao, J.; Han, G.; Liu, Z.; Liu, C.; Zhang, C.; Liu, B.; Jiang, C.; Liu, R.; Zhao, T.; Han, M.-Y.; Zhang, Z. J. Am. Chem. Soc. 2016, 138, 3769.

[8] Chen, X.; Tian, X.; Shin, I.; Yoon, J. Chem. Soc. Rev. 2011, 40, 4783.

[9] (a) Zhang, H.; Liu, R.; Liu, J.; Li, L.; Wang, P.; Yao, S. Q.; Xu, Z.; Sun, H. Chem. Sci. 2016, 7, 256.

(b) Meimetis, L. G.; Giedt, R. J.; Mikula, H.; Carlson, J. C.; Kohler, R. H.; Pirovich, D. B.; Weissleder, R. Chem. Commun. 2016, 52, 9953.

[10] (a) Chen, X.; Lee, K.-A.; Ren, X.; Ryu, J.-C.; Kim, G.; Ryu, J.-H.; Lee, W.-J.; Yoon, J. Nat Protoc. 2016, 11, 1219.

(b) Fan, J.; Han, Z.; Kang, Y.; Peng, X. Sci. Rep. 2016, 6, 19562.

(c) Zhu, X.; Xiong, M.; Liu, H.-W.; Mao, G.-J.; Zhou, L.; Zhang, J.; Hu, X.; Zhang, X.-B.; Tan, W. Chem. Commun. 2016, 52, 733.

[11] (a) Choi, S.-A.; Park, C. S.; Kwon, O. S.; Giong, H.-K.; Lee, J.-S.; Ha, T. H.; Lee, C.-S. Sci. Rep. 2016, 6, 26203.

(b) Li, W.; Fang, B.; Jin, M.; Tian, Y. Anal. Chem. 2017, 89, 2553.

(c) Xu, K.; Luan, D.; Wang, X.; Hu, B.; Liu, X.; Kong, F.; Tang, B. Angew. Chem. 2016, 128, 12943.

[12] (a) Deng, B.; Ren, M.; Wang, J.-Y.; Zhou, K.; Li, W. Sens. Actuators, B 2017, 248, 50.

(b) Cheng, D.; Pan, Y.; Wang, L.; Zeng, Z.; Yuan, L.; Zhang, X.; Chang, Y.-T. J. Am. Chem. Soc. 2017, 139, 285.

(c) Li, Y.; Lü, Z.; Liu, M.; Xing. G. Chin. J. Org. Chem. 2016, 36, 962 (in Chinese).

(李杨洁, 吕子奇, 刘敏, 邢国文, 有机化学, 2016, 36, 962.)

[13] (a) Yuan, Q.; Zhao, Z.-M.; Zhang, Y.-R.; Su, L.; Miao, J.-Y.; Zhao, B.-X. Sens. Actuators, B. 2017, 247, 736.

(b) Wang, Y.; Li, J.; Feng, L.; Yu, J.; Zhang, Y.; Ye, D.; Chen, H.-Y.
Anal. Chem. 2016, 88, 12403.

(c) Li, M.; Wang, Y.; Liu, G.; Lü, H.; Xing, G. Chin. J. Org. Chem. 2017, 37, 356 (in Chinese).

(李美含，王宇童，刘广建，吕海娟，邢国文，有机化学，2017, 37,356 .)

[14] (a) Zhang, J.-X.; Li, H.; Chen, C.-F.; Lan, R.; Chan, W.-L.; Law, G.-L.; Wang, W.-K.; Wang, K.-L. Chem. Commun. 2012, 48, 9646.

(b) Wang, B.; Fan, J.; Wang, X.; Zhu, H.; Wang, J.; Mu, H.; Peng, X. Chem. Commun. 2015, 51, 792 .

(c) Singh, H.; Lee, H. W.; Heo, C. H.; Byun, J. W.; SarKar, A. R.; Kim, H. M. Chem. Commun. 2015, 51, 12099.

[15] (a) Shi, Y.; Lan, F.; Matson, C.; Mulligan, P.; Whetstine, J. R.; Cole, P. A.; Casero, R. A.; Shi, Y. Cell 2004, 119, 941.

(b) Tsukada, Y.-I.; Fang, J.; Erdjument-Bromage, H.; Warren, M. E.; Borchers, C. H.; Tempst, P. and Zhang, Y. Nature 2006, 439, 811.

(c) Whetstine, J. R.; Nottke, A.; Lan, F.; Huarte, M.; Smolikov, S.; Chen, Z.; Spooner, E.; Li, E.; Zhang, G.; Colaiacovo, M. Cell 2006, $125,467$.

[16] Tong, Z.; Han, C.; Luo, W.; Wang, X.; Li, H.; Luo, H.; Zhou, J.; Qi, J.; He, R. Age 2013, 35, 583.

[17] Tong, Z.; Zhang, J.; Luo, W.; Wang, W.; Li, F.; Li, H.; Luo, H.; Lu, J.; Zhou, J.; Wan, Y.; He, R. Neurobiol. Aging 2011, 32, 31.

[18] Tulpule, K.; Dringen, R. J. Neurochem. 2013, 127, 7.

[19] (a) Goodman, C. G.; Johnson, J. S. J. Am. Chem. Soc. 2015, 137, 14574.

(b) Overman, L. E.; Humphreys, P. G.; Welmaker, G. S. Org. React. 2011, 748 .

(c) Winter, R. F.; Rauhut, G. Chem.-Eur. J. 2002, 8, 641 .

(d) Mccormack, M. P.; Shalumova, T.; Tanski, J. M.; Waters, S. P. Org. Lett. 2010, 12, 3906.

[20] Brewer, T. F.; Chang, C. J. J. Am. Chem. Soc. 2015, 137, 10886.

[21] Roth, A.; Li, H.; Anorma, C.; Chan, J. J. Am. Chem. Soc. 2015, 137, 10890.

[22] Xu, J.; Zhang, Y.; Zeng, L.; Liu, J.; Kinsella, J. M.; Sheng, R. Talanta 2016, 160, 645.

[23] He, L.; Yang, X.; Liu, Y.; Kong, X.; Lin, W. Chem. Commun. 2016 52, 4029.

[24] (a) Dai, Z.-R.; Ge, G.-B.; Feng, L.; Ning, J.; Hu, L.-H.; Jin, Q.; Wang, D.-D.; Lv, X.; Dou, T.-Y.; Cui, J.-N. J. Am. Chem. Soc. 2015, 137, 14488

(b) Zhu, B.; Li, P.; Shu, W.; Wang, X.; Liu, C.; Wang, Y.; Wang, Z.; Wang, Y.; Tang, B. Anal. Chem. 2016, 88, 12532.

(c) Zhou, L.; Zhang, X.; Wang, Q.; Lv, Y.; Mao, G.; Luo, A.; Wu, Y.; Wu, Y.; Zhang, J.; Tan, W. J. Am. Chem. Soc. 2014, 136, 9838.

(d) Zhang, J.; Zhu, X.-Y.; Hu, X.-X.; Liu, H.-W.; Li, J.; Feng, L. L.; Yin, X.; Zhang, X.-B.; Tan, W. Anal. Chem. 2016, 88, 11892.

(e) Bae, S. K.; Heo, C. H.; Choi, D. J.; Sen, D.; Joe, E.-H.; Cho, B. R.; Kim, H. M. J. Am. Chem. Soc. 2013, 135, 9915.

(f) Cheng, D.; Pan, Y.; Wang, L.; Zeng, Z.-B.; Yuan, L.; Zhang, X.-B.; Chang, Y.-T. J. Am. Chem. Soc. 2017, 139, 285.

(g) Kim, H. M.; Cho, B. R. Acc. Chem. Res. 2009, 42, 863.

[25] Li, J.-B.; Wang, Q.-Q.; Yuan, L.; Wu, Y.-X.; Hu, X.-X.; Zhang, X.-B.; Tan, W. Analyst 2016, 141, 3395.

[26] Xie, Z.; Ge, J.; Zhang, H.; Bai, T.; He, S.; Ling, J.; Sun, H.; Zhu, Q. Sens. Actuators, B 2017, 241, 1050.

[27] Qian, X.; Xiao, Y.; Xu, Y.; Guo, X.; Qian, J.; Zhu, W. Chem. Commun. 2010, 46, 6418

[28] Ethirajan, M.; Chen, Y.; Joshi, P.; Pandey, R. K. Chem. Soc. Rev. 2011, 40, 340.

[29] Kotagiri, N.; Sudlow, G. P.; Akers, W. J.; Achilefu, S. Nat. Nanotechnol. 2015, 10, 370.

[30] Liu, W.; Truillet, C.; Flavell, R. R.; Brewer, T. F.; Evans, M. J.; Wilson, D. M.; Chang, C. J. Chem. Sci. 2016, 7, 5503.

[31] (a) Han, Q.; Mou, Z.; Wang, H.; Tang, X.; Dong, Z.; Wang, L.; Dong, X.; Liu, W. Anal. Chem. 2016, 88, 7206.

(b) Shu, W.; Yan, L.; Liu, J.; Wang, Z.; Zhang, S.; Tang, C.; Liu, C.; Zhu, B.; Du, B. Ind. Eng. Chem. Res. 2015, 54, 8056.

(c) Zhou, P.; Yao, J.; Hu, G.; Fang, J. ACS Chem. Biol. 2016, 11, 
1098.

(d) Liu, X.-L.; Du, X.-J.; Dai, C.-G.; Song, Q.-H. J. Org. Chem. 2014, 79, 9481.

[32] Tang, Y.; Kong, X.; Liu, Z.-R.; Xu, A.; Lin, W. Anal. Chem. 2016, $88,9359$.

[33] Lee, Y. H.; Tang, Y.; Verwilst, P.; Lin, W.; Kim, J. S. Chem. Commun. 2016, 52, 11247.

[34] (a) Watts, C. Biochim. Biophys. Acta, Proteins Proteomics 2012, $1824,14$. (b) Zhao, H. Traffic 2012, 13, 1307.

[35] Zhu, H.; Fan, J.; Du, J.; Peng, X. Acc. Chem. Res. 2016, 49, 2115.

[36] (a) Zhou, J.; Shi, W.; Li, L.; Gong, Q.; Wu, X.; Li, X.; Ma, H. Anal. Chem. 2016, 88, 4557.

(b) Dong, B.; Song, X.; Wang, C.; Kong, X.; Tang, Y.; Lin, W. Anal. Chem. 2016, 88, 4085.

(c) Qiu, K.; Huang, H.; Liu, B.; Liu, Y.; Huang, Z.; Chen, Y.; Ji, L.-N.; Chao, H. ACS Appl. Mater. Interfaces. 2016, 8, 12702.

(d) Wan, Q.; Chen, S.; Shi, W.; Li, L.; Ma, H. Angew. Chem., Int. Ed. 2014, 53, 10916.

(e) Wang, Y.; Li, J.; Feng, L.; Yu, J.; Zhang, Y.; Ye, D.; Chen, H.-Y. Anal. Chem. 2016, 88, 12403.

(f) Fan, J.; Han, Z.; Kang, Y.; Peng, X. Sci. Rep. 2016, 6, 1.

[37] Tang, Y.; Kong, X.; Xu, A.; Dong, B.; Lin, W. Angew. Chem., Int. Ed. 2016, 55, 3356.

[38] Liu, C.; Shi, C.; Li, H.; Du, W.; Li, Z.; Wei, L.; Yu, M. Sens. Actuators, B 2015, 219, 185.

[39] Song, H.; Rajendiran, S.; Kim, N.; Jeong, S. K.; Koo, E.; Park, G.; Thangadurai, T. D.; Yoon, S. Tetrahedron Lett. 2012, 53, 4913.

[40] Dong, B.; Song, X.; Tang, Y.; Lin, W. Sens. Actuators, B 2016, 222, 325.

[41] Liu, C.; Cheng, A.-W.; Xia, X.-K.; Liu, Y.-F.; He, S.-W.; Guo, X.; Sun, J.-Y. Anal. Methods 2016, 8, 2764.

[42] Aksornneam, L.; Kanatharana, P.; Thavarungkul, P.; Thammakhet, C. Anal. Methods 2016, 8, 1249.

[43] He, L.; Yang, X.; Ren, M.; Kong, X.; Liu, Y.; Lin, W. Chem. Commun. 2016, 52, 9582.

[44] Zhao, X. J.; Yang, J. H.; Li, Y. F. Sens. Actuators, B 2014, 203, 417.

[45] Zhang, S.; Yang, H.; Ma, Y.; Fang, Y. Sens. Actuators, B 2016, 227, 271.

[46] Wong, K.-F.; Deng, J.-R.; Wei, X.-Q.; Shao, S.-P.; Xiang, D.-P.; Wong, M.-K. Org. Biomol. Chem. 2015, 13, 7408.

[47] Lin, J.-M.; Huang, Y.-Q.; Liu, Z.-B.; Lin, C.-Q.; Ma, X.; Liu, J.-M. RSC Adv. 2015, 5, 99944.

[48] Thornalley, P. J. Drug Metabol. Drug Interact. 2008, 23, 125.

[49] (a) Matafome, P.; Sena, C.; Seiça, R. Endocrine 2013, 43, 472. (b) Degen, J.; Vogel, M.; Richter, D.; Hellwig, M.; Henle, T. J. Agric. Food Chem. 2013, 61, 10253.

[50] Kilhovd, B. K.; Juutilainen, A.; Lehto, S.; Rönnemaa, T.; Torjesen, P. A.; Hanssen, K. F.; Laakso, M. Atherosclerosis 2009, 205, 590.

[51] Eberhardt, M. J.; Filipovic, M. R.; Leffler, A.; De La Roche, J.; Kistner, K.; Fischer, M. J.; Fleming, T.; Zimmermann, K.; IvanovicBurmazovic, I.; Nawroth, P. P. J. Biol. Chem. 2012, 287, 28291.

[52] Nakayama, K.; Nakayama, M.; Iwabuchi, M.; Terawaki, H.; Sato, T.; Kohno, M.; Ito, S. Am. J. Nephrol. 2008, $28,871$.

[53] Wang, T.; Douglass Jr, E. F.; Fitzgerald, K. J.; Spiegel, D. A. J. Am. Chem. Soc. 2013, 135, 12429.

[54] (a) Hou, J.-T.; Wu, M.-Y.; Li, K.; Yang, J.; Yu, K.-K.; Xie, Y.-M.; Yu, X.-Q. Chem. Commun. 2014, 50, 8640.

(b) Yuan, H.; Cho, H.; Chen, H. H.; Panagia, M.; Sosnovik, D. E.; Josephson, L. Chem. Commun. 2013, 49, 10361.

(c) Xiao, H.; Li, J.; Zhao, J.; Yin, G.; Quan, Y.; Wang, J.; Wang, R. J. Mater. Chem. B 2015, 3, 1633.

(d) Li, P.; Zhang, W.; Li, K.; Liu, X.; Xiao, H.; Zhang, W.; Tang, B. Anal. Chem. 2013, 85, 9877. (e) Masanta, G.; Heo, C. H.; Lim, C. S.; Bae, S. K.; Cho, B. R.; Kim, H. M. Chem. Commun. 2012, 48, 3518.

(f) Lim, C. S.; Masanta, G.; Kim, H. J.; Han, J. H.; Kim, H. M.; Cho, B. R. J. Am. Chem. Soc. 2011, 133, 11132.

[55] Pun, P. B. L.; Logan, A.; Darley-Usmar, V.; Chacko, B.; Johnson, M. S.; Huang, G. W.; Rogatti, S.; Prime, T. A.; Methner, C.; Krieg, T.; Fearnley, I. M.; Larsen, L.; Larsen, D. S.; Menger, K. E.; Collins, Y.; James, A. M.; Kumar, G. D. K.; Hartley, R. C.; Smith, R. a. J.; Murphy, M. P. Free Radicals Biol. Med. 2014, 67, 437.

[56] Tang, T.; Zhou, Y.; Chen, Y.; Li, M.; Feng, Y.; Wang, C.; Wang, S.; Zhou, X. Anal. Methods 2015, 7, 2386.

[57] Mei, B.; Miao, Q.; Tang, A.; Liang, G. Nanoscale 2015, 7, 15605.

[58] Yang, Z.; Liang, G.; Ma, M.; Abbah, A. S.; Lu, W. W.; Xu, B. Chem. Commun. 2007, 8, 843.

[59] Su, T.; Tang, Z.; He, H.; Li, W.; Wang, X.; Liao, C.; Sun, Y.; Wang, Q. Chem. Sci. 2014, 5, 4204.

[60] Yu, Y.; Chau, Y. Biomacromolecules 2015, 16, 56.

[61] Miao, Q.; Wu, Z.; Hai, Z.; Tao, C.; Yuan, Q.; Gong, Y.; Guan, Y.; Jiang, J.; Liang, G. Nanoscale 2015, 7, 2797.

[62] Liu, S.; Luo, Y.; Liang, G. Nanoscale 2016, 8, 766.

[63] Yuen, L. H.; Saxena, N. S.; Park, H. S.; Weinberg, K.; Kool, E. T. ACS Chem. Biol. 2016, 11, 2312.

[64] Liu, C.; Jiao, X.; He, S.; Zhao, L.; Zeng, X. Dyes Pigm. 2017, 138, 23.

[65] (a) Zhang, F.; Liang, X.; Zhang, W.; Wang, Y.-L.; Wang, H.; Mohammed, Y. H.; Song, B.; Zhang, R.; Yuan, J. Biosens. Bioelectron. 2017, 87, 1005.

(b) Cao, L.; Zhang, R.; Zhang, W.; Du, Z.; Liu, C.; Ye, Z.; Song, B.; Yuan, J. Biomaterials 2015, 68, 21.

(c) Zhang, R.; Ye, Z.; Song, B.; Dai, Z.; An, X.; Yuan, J. Inorg. Chem. 2013, 52, 10325.

(d) Zhang, R.; Ye, Z.; Yin, Y.; Wang, G.; Jin, D.; Yuan, J.; Piper, J. A. Bioconjugate Chem. 2012, 23, 725 .

[66] Zhang, W.; Zhang, F.; Wang, Y.-L.; Song, B.; Zhang, R.; Yuan, J. Inorg. Chem. 2017, 56, 1309.

[67] Gorrini, C.; Harris, I. S. and Mak, T. W. Nat. Rev. Drug Discovery 2013, 12, 931.

[68] Riggins, J. N.; Pratt, D. A.; Voehler, M.; Daniels, J. S.; Marnett, L. J. J. Am. Chem. Soc. 2004, 126, 10571.

[69] Niedernhofer, L. J.; Daniels, J. S.; Rouzer, C. A.; Greene, R. E.; Marnett, L. J. J. Biol. Chem. 2003, 278, 31426.

[70] (a) Jain, S. K.; Mcvie, R.; Smith, T. Diabetes Care 2000, 23, 1389. (b) Tajika, K.; Okamatsu, K.; Takano, M.; Inami, S.; Yamamoto, M.; Murakami, D.; Kobayashi, N.; Ohba, T.; Hata, N.; Seino, Y.; Mizuno, K. Circ. J. 2012, 76, 2211.

(c) Romieu, I.; Barraza-Villarreal, A.; Escamilla-Nuñez, C.; Almstrand, A.-C.; Diaz-Sanchez, D.; Sly, P. D.; Olin, A.-C. J. Allergy Clin. Immunol. 2008, 121, 903.

(d) Dillioglugil, M. O.; Mekık, H.; Muezzinoglu, B.; Ozkan, T. A.; Demir, C. G.; Dillioglugil, O. Int. Urol. Nephrol. 2012, 44, 1691.

[71] Chen, J.; Zeng, L.; Xia, T.; Li, S.; Yan, T.; Wu, S.; Qiu, G.; Liu, Z. Anal. Chem. 2015, 87, 8052.

[72] Pilz, J.; Meineke, I.; Gleiter, C. H. J. Chromatogr. Biomed. Appl. 2000, 742, 315 .

[73] Hensley, K.; Robinson, K. A.; Gabbita, S. P.; Salsman, S.; Floyd, R. A. Free Radic. Biol. Med. 2000, $28,1456$.

[74] (a) Sharmin, S.; Sakata, K.; Kashiwagi, K.; Ueda, S.; Iwasaki, S.; Shirahata, A.; Igarashi, K. Biochem. Biophys. Res. Commun. 2001, 282, 228.

(b) Yoshida, M.; Tomitori, H.; Machi, Y.; Hagihara, M.; Higashi, K.; Goda, H.; Ohya, T.; Niitsu, M.; Kashiwagi, K.; Igarashi, K. Biochem. Biophys. Res. Commun. 2009, 378, 313.

[75] Togashi, M.; Terai, T.; Kojima, H.; Hanaoka, K.; Igarashi, K.; Hirata, Y.; Urano, Y.; Nagano, T. Chem. Commun. 2014, 50, 14946.

(Cheng, F.) 